\title{
Pseudodifferential calculus on a singular foliation
}

\author{
Iakovos Androulidakis and Georges Skandalis*
}

\begin{abstract}
In a previous paper ([1]), we associated a holonomy groupoid and a $\mathrm{C}^{*}$-algebra to any singular foliation $(M, \mathcal{F})$. Using these, we construct the associated pseudodifferential calculus. This calculus gives meaning to a Laplace operator of any singular foliation $\mathcal{F}$ on a compact manifold $M$, and we show that it can be naturally understood as a positive, unbounded, self-adjoint operator on $L^{2}(M)$.
\end{abstract}

Mathematics Subject Classification (2010). Primary 47G30, 57R30; Secondary 46L87.

Keywords. Pseudodifferential operator, singular foliation, $\mathrm{C}^{*}$-algebra.

\section{Introduction}

This paper is a continuation of our previous paper [1]. There we defined the holonomy groupoid of any singular foliation $\mathcal{F}$ on a smooth manifold $M$. Although this groupoid is a rather ill behaved object, we could define

- the convolution algebra $\mathcal{A}(M, \mathcal{F})$ of "smooth compactly supported" functions on this groupoid;

- the full and reduced $\mathrm{C}^{*}$-algebra of the foliation which are suitable (Hausdorff) completions of this convolution algebra.

A key notion in [1] is that of a bi-submersion which will be also of importance here. This is loosely speaking a cover of an open subset of the holonomy groupoid. It is given by a manifold $U$ with two submersions $s, t: U \rightarrow M$, each of which lifts the leaves of $\mathcal{F}$ to the fibers of $s$ and $t$.

Here we proceed and construct the longitudinal pseudodifferential calculus for our foliations.

The longitudinal differential operators are very easily defined: they are generated by vector fields along the foliation.

The longitudinal pseudodifferential operators are obtained as images of distributions on bi-submersions with "pseudodifferential singularities" along a bisection:

*This research was supported in part by Fundação para a Ciência e a Tecnologia (FCT) through the Centro de Matemática da Universidade do Porto 〈www.fc.up.pt/cmup〉. I. A. devotes this work to Odysseas' Oth birthday. 
Let $(U, t, s)$ be a bi-submersion and $V \subset U$ an identity bisection. Denote by $N$ the normal bundle to $V$ in $U$ and let $a$ be a (classical) symbol on $N^{*}$. Let $\chi$ be a smooth function on $U$ supported on a tubular neighborhood of $V$ in $U$ and let $\phi: U \rightarrow N$ be an inverse of the exponential map (defined on the neighborhood of $V$ ). A pseudodifferential kernel on $U$ is a (generalized) function $k_{a}: u \mapsto$ $\int a(p(u), \xi) \exp (i \phi(u) \xi) \chi(u) d \xi$ (here $p: U \rightarrow M$ is the composition $U \stackrel{\phi}{\rightarrow} N \stackrel{q}{\rightarrow}$ $V$, where $q$ is the vector bundle projection $(x, \xi) \mapsto x$, the integral is an oscillatory integral taken over the vector space $\left.N_{p(u)}^{*}\right)$.

The principal symbol of such an operator is a homogeneous function on a locally compact space $\mathscr{F}^{*}$ which is a family of vector spaces (of non constant dimension).

As in the case of foliations and Lie groupoids (cf. [5], [12], [13], [18]), we show:

- The kernel $k_{a}$ defines a multiplier of $\mathcal{A}(M, \mathcal{F})$ (more precisely, of the image of $\mathcal{A}(M, \mathcal{F})$ in the $\mathrm{C}^{*}$-algebra of the foliation).

- Those multipliers form an algebra.

- The algebra of pseudodifferential operators is filtered by the order of $a$. The class of $k_{a}$ only depends up to lower order on the germ of the principal part of $a$ on $\mathcal{F}^{*}$.

- Negative order pseudodifferential operators are elements of the $\mathrm{C}^{*}$-algebra (full and therefore reduced) of the foliation.

- Zero-order pseudodifferential operators define bounded multipliers of the $\mathrm{C}^{*}$ algebra of the foliation.

- We therefore have an exact sequence of $\mathrm{C}^{*}$-algebras

$$
0 \rightarrow C^{*}(M, \mathcal{F}) \rightarrow \Psi^{*}(M, \mathcal{F}) \rightarrow B \rightarrow 0,
$$

where $\Psi^{*}(M, \mathcal{F})$ denotes the closure of the algebra of zero-order pseudodifferential operators and $B$ is (a quotient of) the algebra $C_{0}\left(S^{*} \mathcal{F}\right)$ of continuous functions on the "cosphere bundle" which vanish at infinity.

- Longitudinally elliptic operators of positive order (i.e., operators whose principal symbol is invertible when restricted to $\mathcal{F}^{*}$ ) give rise to regular quasi-invertible operators.

- We may form a Laplacian of $\mathcal{F}$, which is an example of such a positive order longitudinally elliptic operator. It defines a regular positive self-adjoint multiplier of the $\mathrm{C}^{*}$-algebra, and therefore a positive self-adjoint operator in any non-degenerate representation of $C^{*}(\mathscr{F})$; in particular a self-adjoint element of $B\left(L^{2}(M)\right)$.

One can also take coefficients on a smooth vector bundle over $M$. This allows to build an index theory, which we intend to treat in a subsequent paper.

The paper is organized as follows:

In Section 1 we recall basic facts about pseudodifferential calculus: we define distributions on a manifold $U$ with singularities on a submanifold $V$ and state the 
main classical results that will be used in the subsequent sections, namely:

(a) Such distributions have a principal symbol which is a smooth function in the co-shpere bundle of $N^{*}$, where $N$ is the normal bundle of $V$ in $U$.

(b) We discuss pull backs and push forwards (partial integrations) of pseudodifferential distributions.

(c) If $V_{1}, V_{2}$ are transversal to each other then the product of $P_{1} \in \mathcal{P}\left(U, V_{1}\right)$ and $P_{2} \in \mathcal{P}\left(U, V_{2}\right)$ is a well-defined distribution; a partial integral gives rise to an element of $\mathcal{P}\left(W, V_{1} \cap V_{2}\right)$ whose principal symbol is the product of the principal symbols.

(d) The algebra $C_{c}^{\infty}(U)$ is dense in $\mathcal{P}(U, V)$.

In Section 2, for the convenience of the reader, we briefly recall the framework we introduced in [1] and give some slight modifications of results there. We moreover define the "cotangent space" $\mathcal{F}^{*}$ together with its natural locally compact topology.

In Section 3 we define the longitudinal pseudodifferential operators. Namely, we define an algebra $\Psi^{\infty}(\mathcal{U}, \mathcal{V})$ of pseudodifferential operators associated with an atlas of bi-submersions $U$ and a family of identity bisections $\mathcal{V}$ covering $M$.

For this algebra to be defined reasonably, one needs to bear in mind the following: In case the foliation is regular (or defined by a Lie groupoid), the longitudinal pseudodifferential operators form a subalgebra of the multipliers of the groupoid convolution algebra. A general singular foliation may not arise from a Lie groupoid, but it always comes from an atlas of bi-submersions. So, in order for $\Psi^{\infty}(\mathcal{U}, \mathcal{V})$ to generalize properly the pseudodifferential calculus of the regular case, we define its elements a priori as multipliers of the image of the convolution algebra $\mathcal{A} u$ in its Hausdorff completion $C^{*}(\mathcal{U})$.

This is achieved by showing in $\S 3.3$ that every pseudodifferential kernel $P \in$ $\mathcal{P}(U, V)$ defines a multiplier $\tilde{\theta}_{U, V}(P)$ of the image of the natural morphism $\theta: \mathcal{A} u \rightarrow$ $C^{*}(\mathcal{U})$ from $\mathcal{A} u$ to its Hausdorff completion. To this end, we need to show in $\S 3.2$ that a non-degenerate representation $\Pi$ of $C^{*}(\mathcal{U})$ on a Hilbert space $\mathcal{H}$ admits an appropriate extension to compactly supported pseudodifferential kernels.

In $\$ 3.5$ we show that our pseudodifferential operators have a principal symbol which is a homogeneous function on the subset of non-zero elements in $\mathcal{F}^{*}$. Last, in $\S 3.6$, we show that thus defined, pseudodifferential operators form a $*$-algebra.

In Section 4 we show that our longitudinal pseudodifferential calculus has the classical ellipticity properties. Namely the existence of parametrices for elliptic operators and the existence of square roots for even order, self-adjoint operators with positive principal symbol.

In Section 5 we establish the extension

$$
0 \rightarrow C^{*}(M, \mathcal{F}) \rightarrow \Psi^{*}(M, \mathcal{F}) \rightarrow B \rightarrow 0
$$

discussed above. 
In Section 6 we show how our pseudodifferential calculus allows for the Laplacian of a singular foliation to be realized as a self-adjoint element of $B\left(L^{2}(M)\right)$.

\section{Generalized functions with pseudodifferential singularities}

In this section we recall some well known facts on pseudodifferential distributions and operators.

1.1. Symbols. The symbols that we consider are the "classical" or "polyhomogeneous" symbols. Let us briefly recall how they are defined:

- Let $k, n \in \mathbb{N}, V$ be an open subset of $\mathbb{R}^{n}$. For $m \in \mathbb{Z}$, define the space $S^{m}\left(V \times \mathbb{R}^{k}\right)$ of symbols of order (less than or equal to) $m$ to be the set of smooth functions $a: V \times \mathbb{R}^{k} \rightarrow \mathbb{C}$ such that for any compact set $K \subset V$ and any multi-indices $\alpha \in \mathbb{N}^{n}$ and $\beta \in \mathbb{N}^{k}$ there is a constant $C_{K, \alpha, \beta} \in \mathbb{R}_{+}$such that, for all $x \in K$ and $\xi \in \mathbb{R}^{n}$ we have

$$
\left|\partial_{x}^{\alpha} \partial_{\xi}^{\beta} a(x, \xi)\right| \leq C_{K, \alpha, \beta}(1+|\xi|)^{m-|\beta|} .
$$

- A symbol $a \in S^{m}\left(V \times \mathbb{R}^{k}\right)$ is called classical or polyhomogeneous if $a \sim$ $\sum_{k=-\infty}^{m} a_{k}$, where $a_{k}$ are positively homogeneous functions of degree $k$ in the second variable, namely they satisfy $a_{k}(x, t \xi)=t^{k} a(x, \xi)$ for all $\xi \neq 0$ and $t>0$. The notation " $\sim$ " means that $a(x, \xi)-\chi(\xi) \sum_{k=m-M+1}^{m} a_{k}(x, \xi) \in$ $S^{m-M}\left(V \times \mathbb{R}^{k}\right)$ for all $M \in \mathbb{N}$. Here $\chi$ is a cut-off function with $\chi(\xi)=$ 0 if $|\xi|<1 / 2$ and $\chi(\xi)=1$ if $|\xi| \geq 1$. Note that this property does not depend on the cut-off function $\chi$. We will consider only classical symbols in this paper.

- These notions are diffeomorphism invariant and thus allow to define symbols on vector bundles: Given a smooth manifold $V$ and a smooth vector bundle $N$ over $V$, we may define the space of classical symbols $S_{\mathrm{cl}}^{m}(V, N)$ on the bundle $N$ : these are functions $a$ on the total space $N$ which admit an expansion $a \sim \sum_{k=-\infty}^{m} a_{k}$ as above in any chart where the bundle $N$ is trivial.

- We will be mostly interested in the subspace $S_{\mathrm{cl}, \mathrm{c}}^{m}(V, N) \subset S_{\mathrm{cl}}^{m}(V, N)$ of symbols whose support is compact on the $V$ direction, i.e., such that there exists a compact subset $K$ in $V$ with $a(\xi)=0$ whenever $p(\xi) \notin K(p: N \rightarrow V$ is the bundle projection).

- The above definitions extend to give spaces $S_{\mathrm{cl}}^{m}(V, N ; E)$ and $S_{\mathrm{cl}, \mathrm{c}}^{m}(V, N ; E)$ of symbols with values in a smooth vector bundle $E$ over $V$ (considering $E$ as a subbundle of a trivial bundle). 


\subsection{Pseudodifferential generalized functions and submersions}

Remark 1.1 (on densities). In order to make our constructions (which use integration) independent of choices of Lebesgue measures, we use densities everywhere. We just indicate which densities one has to take, with no further explanations most of the time.

1.2.1. Distributions transverse to a submersion. Let $M, N$ be manifolds, $p: N \rightarrow$ $M$ a submersion and $E$ a vector bundle on $M$. Let $P \in C_{\mathrm{c}}^{-\infty}\left(N ; \Omega^{1} \operatorname{ker} d p \otimes p^{*} E\right)$ be a distribution with compact support on $N$. It defines a distribution $p_{!} P \in$ $C_{\mathrm{c}}^{-\infty}(M ; E)$ by a formula $\left\langle p_{!} P, f\right\rangle=\langle P, f \circ p\rangle\left(f \in C^{\infty}\left(M ; \Omega^{1} M \otimes E^{*}\right)\right.$.

Let $F$ be a vector bundle on $N$. A distribution $P \in C^{-\infty}(N ; F)$ on $N$ is said to be transverse to $p$ if for every $f \in C_{\mathrm{c}}^{\infty}\left(N ; \Omega^{1} \operatorname{ker} d p \otimes F^{*}\right)$, the distribution $p_{!}(f \cdot P)$ is smooth on $M$. If $P$ is transverse to $p$, it restricts to a distribution $P^{\prime}$ on $N^{\prime}=p^{-1}\left(M^{\prime}\right)$ for every submanifold $M^{\prime}$ of $M$. The distribution $P^{\prime}$ is obviously transverse to the restriction $p^{\prime}: N^{\prime} \rightarrow M^{\prime}$ of $p$.

\subsubsection{Generalized functions with pseudodifferential singularities}

On a vector bundle. Let $V$ be a smooth manifold and $N$ a smooth vector bundle over $V$. A symbol $a \in S_{\mathrm{cl}, \mathrm{c}}^{m}\left(V, N^{*} ; \Omega^{1} N^{*}\right)$ defines a generalized function of pseudodifferential type on the total space of $N$ which is given by a formal expression (for $u \in N)$

$$
P_{a}(u)=\int_{N_{p(u)}^{*}} a(p(u), \xi) e^{i\langle u, \xi\rangle},
$$

where $p: N \rightarrow V$ is the bundle map and the integral is an "oscillatory integral". This "function" $P_{a}$ makes sense as a distribution on the total space of $N$, i.e., elements of the dual space of the space of smooth functions with compact support on the total space of $N$ (actually smooth sections of a suitable bundle of one densities).

We have, here $k$ is the dimension of the bundle $N$,

$$
\begin{aligned}
\left\langle P_{a}, f\right\rangle & =(2 \pi)^{-k} \int_{V}\left(\int_{N_{x}^{*} \times N_{x}} a(x, \xi) e^{-i\langle u, \xi\rangle} f(u)\right) \\
& =(2 \pi)^{-k} \int_{V}\left(\int_{N_{x}^{*}} a(x, \xi) e^{-i\langle u, \xi\rangle} \hat{f}(\xi)\right) .
\end{aligned}
$$

The distribution $P_{a}$ is transverse to the projection $p: N \rightarrow M$; we may therefore consider $P_{a}$ as a $C^{\infty}(V)$ linear map from $C_{\mathrm{c}}^{\infty}\left(N ; \Omega^{1} N\right) \rightarrow C^{\infty}(V)$ through the formula (for $x \in V$ )

$$
\left\langle P_{a}, f\right\rangle(x)=(2 \pi)^{-k} \int_{N_{x}^{*}} a(x, \xi) \hat{f}(\xi) .
$$


Along a submanifold. Let $U$ be a smooth manifold and $V$ a closed smooth submanifold of $U$. Denote by $N$ the normal bundle to $V$.

We will use the tubular neighborhood construction. Let us briefly fix the notation: $U_{1}$ denotes a neighborhood of $V$ in $U$ and $\phi: U_{1} \rightarrow N$ denotes a local diffeomorphism such that $\phi(v)=(v, 0)$ for $v \in V$, and $d \phi$ restricted to $V$ is the identity in the normal direction. More explicitly, note that $T_{(v, 0)} N=T_{v} V \oplus N_{v}$ for $v \in V$; the above condition means that $d \phi_{v}$ composed with the second projection is the projection $T_{v} U \rightarrow N_{v}=T_{v} U / T_{v} V$.

A generalized function on $U$ with pseudodifferential singularity on $V$ is a generalized function, which far from $V$ is smooth, and near $V$ coincides with a generalized function of pseudodifferential type through a tubular neighborhood construction.

In other words, $P$ is of the form $P=h+\chi \cdot P_{a} \circ \phi$ where

- $\left(U_{1}, \phi\right)$ is a tubular neighborhood construction as above,

- $h \in C^{\infty}(U)$,

- $\chi$ is a smooth "bump" function equal to 1 in a neighborhood of $V$ and to 0 outside $U_{1}$,

- $a \in S_{\mathrm{cl}}^{m}\left(V, N^{*} ; \Omega^{1} N^{*}\right)$ is a (classical) symbol.

Concretely, such a pseudodifferential function is a distribution on $U$ : If $f \in$ $C_{\mathrm{c}}^{\infty}\left(U ; \Omega^{1}(T U)\right)$, we put

$$
\langle P, f\rangle=\int_{U} h(u) f(u)+(2 \pi)^{-k} \int_{N^{*} U_{1}} a(p \circ \phi(u), \xi) \chi(u) f(u) e^{-i\langle\phi(u), \xi\rangle} .
$$

The generalized functions on $U$ with pseudodifferential singularities on $V$ form a vector space that will be denoted by $\mathcal{P}(U, V)$. We denote by $\mathcal{P}_{\mathrm{c}}(U, V)$ those functions that vanish outside a compact subset of $U$ (i.e., that have the form $\chi P$ where $\chi \in C_{\mathrm{c}}^{\infty}(U)$ and $\left.P \in \mathcal{P}(U, V)\right)$.

Example 1.2. Choose a metric on $U$ and thus a trivialization of all densities. Let $X$ be a vector field with compact support on $U$. The map $q_{X}: f \mapsto \int_{V} X f$ is an example of a (pseudo)differential distribution. Note that if $X$ is tangent to $V$, then $\int_{V} X f=-\int_{V} \operatorname{div}(X) f$. In other words, $q_{X}$ only depends up to order zero operators on the image of $X$ in the normal bundle.

Let us state a few facts about these generalized functions that we will use extensively:

- We may extend the construction of pseudodifferential functions and define pseudodifferential sections of any smooth (complex) vector bundle $E$ over $U$. These also give rise to distributions as above, i.e., linear mappings on $C_{\mathrm{c}}{ }^{\infty}\left(U ; \Omega^{1} T U \otimes E^{*}\right)$. We denote by $\mathcal{P}(U, V ; E)$ the space they form, and by $\mathcal{P}_{\mathrm{c}}(U, V ; E)$ the subspace of those with compact support. 
- The space of generalized functions with pseudodifferential singularities does not depend on the choice of $\phi: U \rightarrow N$ with the above requirements.

We immediately deduce:

Proposition 1.3. A pseudodifferential distribution $P \in \mathcal{P}(U, V ; E)$ is transverse to any submersion $p: U \rightarrow M$ which is transverse to $V$.

Notice that the smooth function on $U \backslash V$ associated with a generalized function $P$ does not determine $P$ : if the symbol is a polynomial, then $P$ is differential and is supported by $V$, i.e., vanishes outside $V$.

1.2.3. Density of smooth functions. Let $P \in \mathcal{P}(U, V)$ be given by the formal formula

$$
P(u)=h(u)+(2 \pi)^{-k} \chi(u) \int_{N_{p(u)}^{*}} a(p \circ \phi(u), \xi) e^{-i\langle\phi(u), \xi\rangle} .
$$

Let $\chi_{1}$ be a smooth nonnegative function with compact support on $\mathbb{R}_{+}$which is equal to 1 in a neighborhood of 0 . Put then

$$
P_{n}(u)=h(u)+(2 \pi)^{-k} \chi(u) \int_{N_{p(u)}^{*}} a(p \circ \phi(u), \xi) \chi_{1}(\|\xi\| / n) e^{-i\langle\phi(u), \xi\rangle} .
$$

Then $P_{n} \in C_{\mathrm{c}}^{\infty}(U)$ and converges to $P$ in the topology of $C^{-\infty}$. Further, for every submersion $q: U \rightarrow M^{\prime}$ which is transverse to $V$ and every $f \in C^{\infty}\left(U ; \Omega^{1} \operatorname{ker} d p\right)$, the sequence of $p_{!}\left(f P_{n}\right)$ of smooth functions on $M^{\prime}$ converges to $p_{!}(f P)$ in the topology of $C_{\mathrm{c}}^{\infty}\left(M^{\prime}\right)$.

1.2.4. Principal symbol. A generalized function $P \in \mathcal{P}(U, V)$ of order $m$ with pseudodifferential singularities has a principal symbol. If $P$ is associated with a symbol $a$ of order $m$, then the principal symbol $\sigma_{m}(P)$ of $P$ is the homogeneous part of $a$ of order $m$. It is defined outside the zero section on the total space of $N$ and $\sigma_{m}(P)(x, \xi)$ is a 1-density on $N_{x}^{*}$ (for $x \in V$ and $\xi \in N_{x}^{*}$ non-zero). By choosing smoothly a Euclidean metric of the bundle $N$, it can be defined as an element $\sigma_{m}(P) \in C^{\infty}\left(S^{*} N\right)$, where $S^{*} N$ is the cosphere bundle of $N^{*}$.

Proposition 1.4. We have an exact sequence

$$
0 \rightarrow \Psi^{m-1}(V, U) \rightarrow \Psi^{m}(V, U) \stackrel{\sigma_{m}}{\longrightarrow} C^{\infty}\left(S^{*} N\right) \rightarrow 0 .
$$

Proof. The only thing that has to be proved is that $\sigma_{m}(P)$ only depends on $P$. This is a classical fact (see, e.g., [13]). Let us recall this briefly:

We may assume that $U=N$. Then $P$ defines a $C^{\infty}(V)$-linear map $C_{c}^{\infty}(N) \rightarrow$ $C_{c}^{\infty}(V)$ (using appropriate densities). Let $x \in V$ and $\xi \in N_{x}$ be a non-zero covector. 
Then $\sigma_{m}(x, \xi)=\lim _{\tau \rightarrow+\infty}(i \tau)^{-m} P\left(e^{i \tau \varphi} \chi\right)(x)$ where $\varphi \in C_{\mathrm{c}}^{\infty}(N)$ with derivative $\xi$ at $x \in V$ (the zero point of $N_{x}$ ) along $N_{x}$, and $\chi \in C_{\mathrm{c}}^{\infty}(N)$ is equal to 1 in a neighborhood of $x$.

We may, of course, add bundles into the picture: if $P \in \mathcal{P}(U, V ; E)$ then it follows that $\sigma_{m}(P)(x, \xi) \in \Omega^{1}\left(N_{x}^{*}\right) \otimes E_{x}$ (for $x \in V$ and $\xi \in N_{x}^{*}$ non-zero).

It is easy to see that generalized functions satisfy all the properties of classical pseudodifferential operators. For future reference in the sequel we recall the following one; it is the key ingredient that provides the existence of parametrices for elliptic pseudodifferential operators.

Theorem 1.5. Let $\left(Q_{n}\right)_{n \in \mathbb{N}}$ be a sequence of pseudodifferential functions such that $Q_{n}-Q_{n+1}$ is of order $m-n$. Then there exists a pseudodifferential function $Q$ such that $Q-Q_{n}$ is of order $m-n$ for all $n$.

Example 1.6. The principal symbol of $q_{X}$ in Example 1.2 is $\xi \mapsto i\langle X \mid \xi\rangle$.

\subsection{Pull-back, push-forward, product}

1.3.1. Pull-back (restriction). Let $U$ and $U^{\prime}$ be smooth manifolds and $V \subset U$ a closed submanifold. Let $p: U^{\prime} \rightarrow U$ be a smooth map, transverse to $V$, and put $V^{\prime}=p^{-1}(V)$. It is a submanifold of $U^{\prime}$. Let $P \in \mathcal{P}(U, V)$. Locally (near a point of $V^{\prime}$ ) we may assume $U=V \times \mathbb{R}^{k}, U^{\prime}=V^{\prime} \times \mathbb{R}^{k}$ and $p\left(x^{\prime}, u\right)=\left(p\left(x^{\prime}\right), u\right)$ for $x^{\prime} \in V^{\prime}$ and $u \in \mathbb{R}^{k}$.

We may then assume that $P$ is given (formally) (see eq. (1.1))) by the formula

$$
P(x, u)=(2 \pi)^{-k} \int_{\mathbb{R}^{k}} e^{-i\langle u, \xi\rangle} a(x, \xi)+h(x, u),
$$

where $h$ is smooth and $a$ is a symbol.

We then define $p^{*} P$ setting $\left(p^{*} P\right)\left(x^{\prime}, u\right)=P\left(p\left(x^{\prime}\right), u\right)$. Under the identification of the normal bundle $N^{\prime}$ of $V^{\prime}$ in $U^{\prime}$ with $p^{*} N$, the principal symbol of $p^{*} P$ is given by $\sigma_{m}\left(p^{*} P\right)=\sigma_{m}(P) \circ p$.

\subsubsection{Push-forward (partial integration)}

Proposition 1.7 (Push-forward). Let $U$ and $U^{\prime}$ be smooth manifolds and $V \subset U$ a closed submanifold. Let $p: U \rightarrow U^{\prime}$ be a submersion which restricts to a diffeomorphism $p: V \rightarrow V^{\prime}$ where $V^{\prime}$ is a submanifold of $U^{\prime}$. Let $E^{\prime}$ be a vector bundle on $U^{\prime}$.

(a) Integration along the fibers of $p$ gives rise to a map $p_{!}: \mathcal{P}_{\mathrm{c}}\left(U, V ; \Omega^{1} \operatorname{ker} d p \otimes\right.$ $\left.p^{*} E^{\prime}\right) \rightarrow \mathcal{P}_{c}\left(U^{\prime}, V^{\prime} ; E^{\prime}\right)$ that is defined by $\left\langle p_{!}(P), f\right\rangle=\langle P, f \circ p\rangle$ for $f \in$ $C^{\infty}\left(U ; \Omega^{1} T U \otimes E^{*}\right)$. The principal symbol of $p_{!} P$ is $\sigma_{m}^{\prime}(x, \xi)=\sigma_{m}\left(x, p^{*} \xi\right)$, where $x \in V^{\prime} \simeq V$ and $p^{*}$ is the (injective) map $\left(T_{x} U^{\prime} / T_{x} V\right)^{*} \rightarrow\left(T_{x} U / T_{x} V\right)^{*}$ induced by $p$. 
(b) If $p$ is onto, then $p$ ! is onto, too.

Proof. (a) We may assume that $p: V \times \mathbb{R}^{k} \times \mathbb{R}^{\ell} \rightarrow V \times \mathbb{R}^{k}$ is the projection and that $P$ is given by the formula

$$
\langle P, f\rangle=(2 \pi)^{-(k+\ell)} \int e^{-i(\langle u, \xi\rangle+\langle v, \eta\rangle)} a(x, \xi, \eta) \chi_{1}(u) \chi_{2}(v) f(x, u, v) .
$$

We thus get

$$
\begin{aligned}
\left\langle p_{!} P, f\right\rangle & =\langle P, f \circ p\rangle \\
& =(2 \pi)^{-(k+\ell)} \int_{V \times \mathbb{R}^{\ell} \times\left(\mathbb{R}^{k+\ell}\right)^{*}} a(x, \xi, \eta) e^{-i\langle u, \xi\rangle} \chi_{1}(u) \widehat{\chi_{2}}(\eta) f(x, u) .
\end{aligned}
$$

Using a Taylor expansion of the form

$$
a(x, \xi, \eta) \sim a(x, \xi, 0)+\sum_{1 \leq|\alpha|} \frac{\eta^{\alpha}}{\alpha !} \frac{\partial^{|\alpha|}}{(\partial \eta)^{\alpha}} a(x, \xi, 0)
$$

we find the principal term

$$
(2 \pi)^{-(k+\ell)} \int_{V \times \mathbb{R}^{\ell} \times\left(\mathbb{R}^{k+\ell}\right)^{*}} a(x, \xi, 0) e^{-i\langle u, \xi\rangle} \chi_{1}(u) \widehat{\chi_{2}}(\eta) f(x, u) .
$$

Since $(2 \pi)^{-\ell} \int \widehat{\chi_{2}}(\eta)=\chi_{2}(0)=1$ we find

$$
(2 \pi)^{-k} \int_{V \times \mathbb{R}^{\ell} \times\left(\mathbb{R}^{k}\right)^{*}} a(x, \xi, 0) e^{-i\langle u, \xi\rangle} \chi_{1}(u) f(x, u) .
$$

(b) Let $P^{\prime} \in \mathcal{P}_{c}\left(U^{\prime}, V^{\prime} ; E^{\prime}\right)$. One obviously may extend the principal symbol of $P^{\prime}$ to get a homogeneous section on the normal bundle of $V$ in $U$. It follows that there exists an operator $P_{1} \in \mathcal{P}_{\mathrm{c}}\left(U, V ; p^{*} E^{\prime}\right)$ such that $p_{!} P_{1}-P^{\prime}$ is of order $m-1$. Using induction, one constructs a sequence $P_{n} \in \mathcal{P}_{\mathrm{c}}\left(U, V ; p^{*} E^{\prime}\right)$ such that $p_{!} P_{n}-P^{\prime}$ is of order $m-n$ and $P_{n+1}-P_{n}$ is of order $m-n$. Using Theorem 1.5, one then gets $Q$ such that $Q-P_{n}$ is of order $m-n$, whence $p_{!} Q-P^{\prime}$ is smoothing. Finally, using partitions of the identity, it is obvious that $p_{!}: C_{\mathrm{c}}^{\infty}\left(U ; p^{*} E^{\prime}\right) \rightarrow C_{\mathrm{c}}\left(U^{\prime}, E^{\prime}\right)$ is onto.

Remarks 1.8. (a) We will also need a slightly more general statement: In the above proposition, we may just assume that $p$ induces a submersion $p: V \rightarrow V^{\prime}$ where $V^{\prime}$ is a submanifold of $U^{\prime}$. In that case, for $P \in \mathcal{P}\left(U, V ; \Omega^{1}(\operatorname{ker} d p) \otimes p^{*}\left(E^{\prime}\right)\right)$, the principal symbol $\sigma^{\prime}\left(x^{\prime}, \xi^{\prime}\right)$ of $p_{!} P$ is the integral of $\sigma\left(x, p_{x}^{*}\left(\xi^{\prime}\right)\right)$ for $x$ running in the fiber $V \cap p^{-1}\left(x^{\prime}\right)$ (and $p_{x}^{*}:\left(T_{x^{\prime}} U^{\prime} / T_{x^{\prime}} V^{\prime}\right)^{*} \rightarrow\left(T_{x} U / T_{x} V\right)^{*}$ is the (injective) map induced by $\left.(d p)_{x}\right)^{1}$

\footnotetext{
${ }^{1}$ An easy check shows that the densities match correctly.
} 
To establish this, one may assume that $U^{\prime}=V^{\prime} \times \mathbb{R}^{k}, U=V^{\prime} \times \mathbb{R}^{j} \times \mathbb{R}^{k} \times \mathbb{R}^{\ell}$, $V=V^{\prime} \times \mathbb{R}^{j} \times\{(0,0)\}$ and that $p$ is the obvious projection $V^{\prime} \times \mathbb{R}^{j} \times \mathbb{R}^{k} \times \mathbb{R}^{\ell} \rightarrow$ $V^{\prime} \times \mathbb{R}^{k}$.

(b) Obviously, we have $(q \circ p) !=q ! \circ p$ ! if $p: U \rightarrow U^{\prime}$ and $q: U^{\prime} \rightarrow U^{\prime \prime}$ are submersions satisfying requirements of (a).

1.3.3. Products. In order to understand the product of pseudodifferential operators in our context, we give the following lemma. We say that a submersion $p: U \rightarrow U^{\prime}$ is strictly transverse to a submanifold $V \subset U$ if at any point $x \in V$ we have $T_{x} V \oplus \operatorname{ker} d p_{x}=T_{x} U$.

Lemma 1.9. Let $U$ be a manifold, let $V_{1}, V_{2}$ be two closed submanifolds of $U$ that are transverse to each other, and let $p: U \rightarrow U^{\prime}$ be a submersion strictly transverse to both $V_{1}$ and $V_{2}$. Then there are charts of $U$ of the form $W \times \mathbb{R}^{k} \times \mathbb{R}^{k}$ covering $V=V_{1} \cap V_{2}$, such that $V_{1}=W \times \mathbb{R}^{k} \times\{0\}, V_{2}=W \times\{0\} \times \mathbb{R}^{k}$ and $p$ can be written as $(v, \xi, \eta) \mapsto(v, \xi+\eta)$.

Proof. Notice that $V$ is a manifold due to the transversality of $V_{1}$ to $V_{2}$. Then $V$ is covered by charts such that $U$ can be written as $U^{\prime} \times T$, where $T=\mathbb{R}^{k}$ is the fiber of $p$ and $p$ is the projection. Since $T$ is transverse to $V_{1}$, for this chart we can write $V_{1}=U^{\prime} \times\{0\}$. Since $V_{2}$ is transversal to $V_{1}$, it is the graph of a submersion $q: V_{1} \rightarrow T$; we have $V=q^{-1}(\{0\})$, hence we can write $V_{1}=V \times \mathbb{R}^{k}$ and $q$ is the projection. Under these identifications, we find a chart $V \times \mathbb{R}^{k} \times \mathbb{R}^{k}$, for which $V_{1}=V \times \mathbb{R}^{k} \times\{0\}, V_{2}=\{(x, \xi, \eta) ; \xi=\eta\}$ and $p(x, \xi, \eta)=\xi$. The result follows by applying the diffeomorphism $(v, \xi, \eta) \mapsto(v, \xi+\eta, \eta)$ on $V \times \mathbb{R}^{k} \times \mathbb{R}^{k}$.

Proposition 1.10. Let $U$ be a manifold and $V_{1}, V_{2}$ two closed submanifolds of $U$ that are transverse to each other. Let $P_{i} \in \mathcal{P}\left(U, V_{i}\right)$.

(a) The product $P_{1} \cdot P_{2}$ makes sense as a distribution on $U$.

(b) Assume that $P_{1} \cdot P_{2}$ has compact support. Let $p: U \rightarrow U^{\prime}$ be a submersion which is both strictly transverse to $V_{1}$ and $V_{2}$ and whose restriction to $V_{1} \cap V_{2}$ is injective and proper. Then $p_{!}\left(P_{1} \cdot P_{2}\right)$ is a pseudodifferential function in $\mathcal{P}\left(U^{\prime}, p\left(V_{1} \cap V_{2}\right)\right)$. If $\sigma_{i}$ is the principal symbol of $P_{i}(i=1,2)$, then the principal symbol of $p_{!}\left(P_{1} . P_{2}\right)$ is $p_{!}\left(\sigma_{1}\right) \cdot p_{!}\left(\sigma_{2}\right)$.

Proof. (a) The statement is local. We may therefore assume that $U=V \times \mathbb{R}^{k} \times \mathbb{R}^{\ell}$, $V_{1}=V \times \mathbb{R}^{k} \times\{0\}$ and $V_{2}=V \times\{0\} \times \mathbb{R}^{\ell}$. Also, by an obvious choice of the tubular neighborhood construction, we may write

and

$$
P_{1}(x, u, v)=\int e^{i\langle v \mid \eta\rangle} a_{1}(x, u, \eta) d \eta
$$

$$
P_{2}(x, u, v)=\int e^{i\langle u \mid \xi\rangle} a_{2}(x, \xi, v) d \xi .
$$


Here $a_{1}$ and $a_{2}$ are classical (polyhomogeneous) symbols.

The product is then given by the formula

$$
\left\langle P_{1} \cdot P_{2}, f\right\rangle=\int\left(\int e^{i\langle u \mid \xi\rangle} e^{i\langle v \mid \eta\rangle} a_{1}(x, u, \eta) a_{2}(x, \xi, v) f(x, u, v) d u d v\right) d x d \xi d \eta,
$$

which makes perfect sense when $f \in C_{\mathrm{c}}^{\infty}(U)$.

(b) Due to Lemma 1.9 we can that assume $U=V \times \mathbb{R}^{k} \times \mathbb{R}^{k}, U^{\prime}=V \times \mathbb{R}^{k}$ and $p(x, u, v)=(x, u+v)$.

We thus have to compute

$$
\left(\int\left(\int e^{i\langle u \mid \xi\rangle} e^{i\langle v \mid \eta\rangle} \chi(x, u, v) a_{1}(x, u, \eta) a_{2}(x, \xi, v) f(x, u+v) d u d v\right) d \xi d \eta\right) d x .
$$

This is a "classical" oscillatory integral on $\mathbb{R}^{4 k}$ which is treated by the usual integration by parts methods. It actually amounts to composing (families indexed by $V$ of) pseudodifferential operators in $\mathbb{R}^{k}$.

\section{Singular foliations, cotangent space}

2.1. Foliations, bi-submersions, atlas, *-algebra. Let us first recall some definitions, notation and conventions used in [1].

\subsubsection{Foliations}

Definition 2.1. (a) Let $M$ be a smooth manifold. A foliation on $M$ is a locally finitely generated submodule of $C_{\mathrm{c}}^{\infty}(M ; T M)$ stable under Lie brackets.

(b) There is an obvious notion of a pull-back foliation: if $(M, \mathcal{F})$ is a foliation and $f: L \times M \rightarrow M$ is the second projection, the pull back foliation $f^{-1}(\mathscr{F})$ is the space of vector fields whose $M$ component as a map from $L$ to $C_{\mathrm{c}}^{\infty}(M, T M)$ takes its values in $\mathscr{F}$. In the same way, one defines pull back foliations by submersions. See [1], Section 1.2.3.

(c) For $x \in M$, put $I_{x}=\left\{f \in C^{\infty}(M): f(x)=0\right\}$. The fiber of $\mathscr{F}$ is the quotient $\widetilde{F}_{x}=\mathscr{F} / I_{x} \mathscr{F}$. The tangent space of the leaf is the image $F_{x}$ of the evaluation map ev $x_{x}: \mathscr{F}^{\rightarrow} \rightarrow T_{x} M$.

The spaces $F_{x}$ and $\mathscr{F}_{x}$ differ on singular leaves: the dimension of $F_{x}$ is lower semi-continuous and the dimension of $\mathscr{F}_{x}$ is upper semi-continuous. They coincide in a dense open subset of $M$, namely on points $x$ that lie in a regular leaf.

We get a surjective linear map $e_{x}: \mathscr{F}_{x} \rightarrow F_{x}$ whose kernel is a Lie algebra $\mathfrak{g}_{x}$.

2.1.2. Bi-submersions, bisections. The main ingredient in the constructions of [1] is the notion of bi-submersion that we now recall. 
Definition 2.2. A bi-submersion of $(M, \mathcal{F})$ is a smooth manifold $U$ endowed with two smooth maps $s, t: U \rightarrow M$ which are submersions and satisfy:

(a) $s^{-1}(\mathscr{F})=t^{-1}(\mathscr{F})$.

(b) $s^{-1}(\mathcal{F})=C_{\mathrm{c}}^{\infty}(U ; \operatorname{ker} d s)+C_{\mathrm{c}}^{\infty}(U ; \operatorname{ker} d t)$.

If $\left(U, t_{U}, s_{U}\right)$ and $\left(V, t_{V}, s_{V}\right)$ are bi-submersions then $\left(U, s_{U}, t_{U}\right)$ is a bi-submersion, as well as $\left(W, s_{W}, t_{W}\right)$ where $W=U \times_{s_{U}, t_{V}} V, s_{W}(u, v)=s_{V}(v)$ and $t_{W}(u, v)=$ $t_{U}(u)$ ([1], Prop. 2.4).

The bi-submersion $\left(U, s_{U}, t_{U}\right)$ is called the inverse of $\left(U, t_{U}, s_{U}\right)$ and is noted $\left(U, t_{U}, s_{U}\right)^{-1}$, or just $U^{-1}$; the bi-submersion $\left(W, s_{W}, t_{W}\right)$ is called the composition of $\left(U, t_{U}, s_{U}\right)$ and $\left(V, t_{V}, s_{V}\right)$ and is denoted by $\left(U, t_{U}, s_{U}\right) \circ\left(V, t_{V}, s_{V}\right)$, or just $U \circ V$.

In [1], Prop. 2.10, it is shown that there are enough bi-submersions $\left\{\left(U_{i}, t_{i}, s_{i}\right)\right\}_{i \in I}$ such that $\bigcup_{i \in I} s_{i}\left(U_{i}\right)=M$ : For an $x \in M$, if $X_{1}, \ldots, X_{n} \in \mathcal{F}$ form a base of $\mathcal{F}_{x}$ then we can find a neighborhood $U$ of $(x, 0)$ in $M \times \mathbb{R}^{n}$ where the exponential $t_{U}(y, \xi)=\exp \left(\sum_{i=1}^{n} \xi_{i} X_{i}\right)(y)$ is defined and such that $\left(U, t_{U}, s_{U}\right)$ is a bi-submersion, where $s_{U}$ denotes the first projection. Such a bi-submersion is sometimes called an identity bi-submersion.

Definition 2.3 (Morphisms of bi-submersions). Let $\left(U_{i}, t_{i}, s_{i}\right), i=1,2$, be bisubmersions. A smooth map $f: U_{1} \rightarrow U_{2}$ is a morphism of bi-submersions if $s_{1}=s_{2} \circ f$ and $t_{1}=t_{2} \circ f$.

In order to compare bi-submersions, we used the notion of bisections:

Definition 2.4. A bisection of $(U, t, s)$ is a locally closed submanifold $V$ of $U$ such that $s$ and $t$ restricted to $V$ are diffeomorphisms to open subsets of $M$. We say that $V$ is an identity bisection if moreover the restrictions of $s$ and $t$ to $V$ coincide.

We say that $u \in U$ carries the foliation-preserving local diffeomorphism $\phi$ if there exists a bisection $V$ such that $u \in V$ and $\phi=\left.t\right|_{V} \circ\left(\left.s\right|_{V}\right)^{-1}$.

In [1], $\$ 2.3$, it is shown that if $\left(U_{j}, t_{j}, s_{j}\right), j=1,2$, are bi-submersions, then a $u_{1} \in U_{1}$ carries the same diffeomorphism with $u_{2} \in U_{2}$ if and only if there exists a morphism of bi-submersions $g$ defined in an open neighborhood of $u_{1} \in U_{1}$ such that $g\left(u_{1}\right)=u_{2}$.

Actually the proof given in [1], §2.3, proves a stronger statement which will be useful here:

Proposition 2.5. Let $\left(U_{j}, t_{j}, s_{j}\right)$ be bi-submersions, $j=1,2, V_{i} \subset U_{i}$ identity bisections and $u_{j} \in V_{j}$ such that $s_{1}\left(u_{1}\right)=s_{2}\left(u_{2}\right)$. Then there exists a morphism of bi-submersions $g$ defined in an open neighborhood $U_{1}^{\prime}$ of $u_{1} \in U_{1}$ such that $g\left(u_{1}\right)=u_{2}$ and $g\left(V_{1} \cap U_{1}^{\prime}\right) \subset V_{2}$. 


\subsubsection{Minimal bi-submersions}

Definition 2.6. If $(U, t, s)$ is a bi-submersion and $u \in U$, then the dimension of the manifold $U$ is at least $\operatorname{dim} M+\operatorname{dim} \mathcal{F}_{s(u)}$. We say that $(U, t, s)$ is minimal at $u$ if $\operatorname{dim} U=\operatorname{dim} M+\operatorname{dim} \widetilde{F}_{s(u)}$.

If $f:\left(U^{\prime}, t^{\prime}, s^{\prime}\right) \rightarrow(U, t, s)$ is a morphism of bi-submersions and $U$ is minimal at $f\left(u^{\prime}\right)$, then $d f_{u^{\prime}}$ is onto. Therefore, there is a neighborhood $W^{\prime}$ of $u^{\prime}$ in $U^{\prime}$ such that the restriction of $f$ to $W^{\prime}$ is a submersion.

For every bi-submersion $(U, t, s)$ and every $u \in U$, there exists a bi-submersion $\left(U^{\prime}, t^{\prime}, s^{\prime}\right)$, and $u^{\prime} \in U^{\prime}$ such that $U^{\prime}$ is minimal at $u^{\prime}$ and carries at $u^{\prime}$ the same diffeomorphisms as $U$ at $u$. It follows that there is a neighborhood $W$ of $u$ in $U$ and a submersion which is a morphism $f:\left(W, t_{\mid W}, s_{\mid W}\right) \rightarrow\left(U^{\prime}, t^{\prime}, s^{\prime}\right)$.

The following result will be used in the sequel.

Proposition 2.7. Let $(U, t, s)$ be a bi-submersion and $V$ an identity bisection. Let $u \in V$ and assume $U$ is minimal at $u$. Then there is a neighborhood $U^{\prime}$ of $u$ in $U$ and a submersion $p: U^{\prime} \circ U^{\prime} \rightarrow U$ which is a morphism of bi-submersions strictly transverse to $U \circ V$ and to $V \circ U$.

Proof. The composition $U \circ U$ carries at $(u, u)$ the identity bisection $V \circ V$. It follows that there exists a neighborhood of $(u, u)$ in $U \circ U$ that we may assume of the form $U^{\prime} \circ U^{\prime}$ and a morphism $p: U^{\prime} \circ U^{\prime} \rightarrow U$. By minimality of $U$ at $u$, we may assume that $p$ is a submersion. Moreover $U \circ V$ and $V \circ U$ are bi-submersions. Again, by minimality of $U$ at $u$, it follows that, up to restricting $U^{\prime}$, the restrictions of $p$ to $(U \circ V) \cap\left(U^{\prime} \circ U^{\prime}\right)$ and $(V \circ U) \cap\left(U^{\prime} \circ U^{\prime}\right)$ are submersions, hence $p$ is transverse to $U \circ V$ and to $V \circ U$, strictly by equality of dimensions.

\subsubsection{Atlas of bi-submersions}

Definition 2.8. Let $\mathcal{U}=\left(\left(U_{i}, t_{i}, s_{i}\right)\right)_{i \in I}$ be a family of bi-submersions. A bisubmersion $(U, t, s)$ is adapted to $U$ if for all $u \in U$ there exists an open subset $U^{\prime} \subset U$ containing $u$, an $i \in I$, and a morphism of bi-submersions $U^{\prime} \rightarrow U_{i}$.

We say that $\mathcal{U}$ is an atlas if

(a) $\bigcup_{i \in I} s_{i}\left(U_{i}\right)=M$,

(b) the inverse of every element in $u$ is adapted to $u$,

(c) the composition $U \circ V$ of any two elements in $U$ is adapted to $U$.

An atlas $\mathcal{V}=\left\{\left(V_{j}, t_{j}, s_{j}\right)\right\}_{j \in J}$ is adapted to $\mathcal{U}$ if every element of $\mathcal{V}$ is adapted to $\mathcal{U}$. We say $\mathcal{U}$ and $\mathcal{V}$ are equivalent if they are adapted to each other. There is a minimal atlas which is adapted to any other atlas: this is the atlas generated by "identity bi-submersions". 
2.1.5. The groupoid of an atlas. The groupoid of an atlas $U=\left(\left(U_{i}, t_{i}, s_{i}\right)\right)_{i \in I}$ is the quotient of $U=\bigsqcup_{i \in I} U_{i}$ by the equivalence relation for which $u \in U_{i}$ is equivalent to $u^{\prime} \in U_{j}$ if $U_{i}$ carries at $u$ the same local diffeomorphisms as $U_{j}$ at $u^{\prime}$.

For every bi-submersion $U$ adapted to $U$ we have a well-defined (quotient) map $\zeta_{U}: U \rightarrow \mathscr{E}_{u}$.

2.1.6. The $\mathbf{C}^{*}$-algebra of a foliation. In [1], $\S 4.3$, we associated to an atlas $U$ a *-algebra $\mathcal{A}(\mathcal{U})=\bigoplus_{i \in I} C_{\mathrm{c}}^{\infty}\left(U_{i} ; \Omega^{1 / 2} U_{i}\right) / \mathcal{I}$. Here $\Omega^{1 / 2}$ denotes the bundle of half densities on $\operatorname{ker} d s \oplus \operatorname{ker} d t$ and $\mathcal{I}$ is the space spanned by the $p_{!}(f)$, where $p: W \rightarrow U$ is a submersion which is a morphism of bi-submersions and $f \in$ $C_{\mathrm{c}}\left(W ; \Omega^{1 / 2} W\right)$ is such that there exists a morphism $q: W \rightarrow V$ of bi-submersions which is a submersion and such that $q !(f)=0$.

We can associate a linear map $Q_{V}: C_{\mathrm{c}}^{\infty}\left(V ; \Omega^{1 / 2} V\right) \rightarrow \mathcal{A} u$ to any bi-submersion $V$ adapted to $\mathcal{U}$. Involution and convolution in $\mathcal{A} u$ are then defined by

$$
\left(Q_{V_{1}}\left(f_{1}\right)\right)^{*}=\left(Q_{V_{1}^{-1}}\right)\left(f_{1}^{*}\right) \quad \text { and } \quad Q_{V_{1}}\left(f_{1}\right) Q_{V_{2}}\left(f_{2}\right)=Q_{V_{1} \circ V_{2}}\left(f_{1} \otimes f_{2}\right)
$$

for $\left(V_{i}, t_{i}, s_{i}\right)$ bi-submersions adapted to $\mathcal{U}$ and $f_{i} \in C_{\mathrm{c}}^{\infty}\left(V_{i} ; \Omega^{1 / 2} V_{i}\right), i=1,2$.

The $C^{*}$-algebra $C^{*}(U)$ of the atlas $U$ is the (Hausdorff) completion of $\mathcal{A} u$ with a natural $C^{*}$-norm [1], $\S \S 4.4-4.5$. Actually, two natural completions were considered: the full and the reduced one.

If $\mathcal{U}$ is adapted to $\mathcal{V}$, we have natural $*$-morphisms $\mathcal{A}(\mathcal{U}) \rightarrow \mathcal{A}(\mathcal{V})$ and $C^{*}(\mathcal{U}) \rightarrow$ $C^{*}(\mathcal{V})$.

When $U$ is the minimal atlas we write $C^{*}(M, \mathcal{F})$ for the full and $C_{r}^{*}(M, \mathcal{F})$ for the reduced completion.

The representations of the full $C^{*}$-algebras were described in [1], $\S 5$, in terms of representations of the associated groupoid. We will come back to this description below (\$3.2).

2.2. The cotangent space. The symbols should be functions on the total space of a "cotangent bundle". Let us discuss this space.

Definition 2.9. The cotangent bundle of the foliation $\mathcal{F}$ (although it is in general not a bundle) is the union $\mathscr{F}=\bigsqcup_{x \in M} \mathscr{F}_{x}^{*}$. It is endowed with a natural projection $p: \mathscr{F}^{*} \rightarrow M((x, \xi) \mapsto x)$. Also, for each $X \in \mathcal{F}$, we have a natural map $q_{X}:(x, \xi) \mapsto \xi \circ e_{x}(X)$. We endow $\mathcal{F}^{*}$ with the weakest topology for which the maps $p$ and $q_{X}$ are continuous.

Proposition 2.10. The space $\mathcal{F}^{*}$ is locally compact.

Proof. It is enough to show that $p^{-1}(U)$ is locally compact for every small enough open set $U$ of $M$. We may therefore assume that $\mathcal{F}$ is finitely generated, i.e., it is a quotient of $C_{\mathrm{c}}^{\infty}\left(M ; \mathbb{R}^{n}\right)$. Then $\mathscr{F}^{*}$ consists of elements of $(x, y) \in M \times\left(\mathbb{R}^{n}\right)^{*}$ 
such that the map $C_{\mathrm{c}}^{\infty}\left(M ; \mathbb{R}^{n}\right) \ni \varphi \mapsto\langle y, \varphi(x)\rangle$ factors through the quotient $\mathcal{F}$ of $C_{\mathrm{c}}^{\infty}\left(M ; \mathbb{R}^{n}\right)$ (i.e., vanishes on the kernel of the map $\left.C_{\mathrm{c}}^{\infty}\left(M ; \mathbb{R}^{n}\right) \rightarrow \mathcal{F}\right)$. It is a closed subset of $M \times\left(\mathbb{R}^{n}\right)^{*}$ and is therefore locally compact.

Example 2.11. Let $\mathcal{F}$ be the foliation on $\mathbb{R}^{3}$ defined by the (infinitesimal) action of $\mathrm{SO}(3)$. It is easy to see that $\mathcal{F}^{*}=\bigcup_{\xi \in \mathbb{R}^{3}}\left\{x \in \mathbb{R}^{3}:\langle x \mid \xi\rangle=0\right\}$.

Let $(U, t, s)$ be a bi-submersion and $V$ an identity bisection. Identifying the normal bundle $N$ with ker $d s$ (or ker $d t$ ), there are epimorphisms $d_{x} t: N_{x} \rightarrow \mathscr{F}_{x}$, $x \in V\left(\right.$ or $\left.d_{x} s\right)$. Dualizing these maps we find that locally the cotangent bundle $\mathscr{F}^{*}$ is a closed subspace of $N^{*}$. Thus we can restrict symbols to $\mathscr{F}^{*}$.

Let $\left(U^{\prime}, t^{\prime}, s^{\prime}\right)$ and $(U, t, s)$ be bi-submersions with identity bisections $V^{\prime}$ and $V$. Let $p: U^{\prime} \rightarrow U$ be a smooth map that is a morphism of bi-submersions such that $p\left(V^{\prime}\right) \subseteq V$. Then for every $x \in s^{\prime}\left(V^{\prime}\right)$ the inclusion $\mathcal{F}_{x}^{*} \rightarrow\left(N_{x}^{\prime}\right)^{*}$ factors as the composition of $p_{x}^{*}: N_{x}^{*} \rightarrow\left(N_{x}^{\prime}\right)^{*}$ with the inclusion $\mathcal{F}_{x}^{*} \rightarrow N_{x}^{*}$.

\section{The space of pseudodifferential kernels on a foliation}

3.1. Pseudodifferential kernels on a bi-submersion. Let $(U, t, s)$ be a bi-submersion. The bundle (over $U$ ) of half densities on $\operatorname{ker} d s \oplus \operatorname{ker} d t$ will be simply denoted by $\Omega^{1 / 2}$.

We define $\mathcal{P}^{m}\left(U, V ; \Omega^{1 / 2}\right)$ (resp. $\left.\mathcal{P}_{c}^{m}\left(U, V ; \Omega^{1 / 2}\right)\right)$ to be the space of generalized sections of the bundle $\Omega^{1 / 2}$ with pseudodifferential singularities along $V$ (resp. those with compact support) of order $\leq m$. We drop the superscript $m$ when we make no order requirements.

Let $N$ be the normal bundle of the inclusion $V \rightarrow U$. Note that $N$ canonically identifies with both the restrictions to $V$ of the bundles ker $d s$ and ker $d t$. Under these identifications the bundle $\Omega^{1} N^{*} \otimes \Omega^{1 / 2}(\operatorname{ker} d s) \otimes \Omega^{1 / 2}(\operatorname{ker} d t)$ is trivial.

The principal symbol of $P \in \mathcal{P}^{m}\left(U, V ; \Omega^{1 / 2}\right)$ is thus an element of $S^{m}\left(V, N^{*}\right)$, i.e., a function on $N^{*}$ which is homogeneous of degree $m$.

Definition 3.1. Let $(U, t, s)$ be a bi-submersion and $V$ an identity bisection.

(a) Denote by $U^{-1}$ the inverse bi-submersion and $\kappa: U \rightarrow U^{-1}$ the (identity) isomorphism. For an operator $P \in \mathcal{P}_{\mathrm{c}}\left(U, V ; \Omega^{1 / 2}\right)$ define $P^{*}=\overline{\kappa ! P}$.

(b) Finally, let $U, U^{\prime}$ be bi-submersions, let $V \subset U$ be an identity bisection, and let $P \in \mathcal{P}_{\mathrm{c}}\left(U, V ; \Omega^{1 / 2}\right)$ and $f \in C_{\mathrm{c}}^{\infty}\left(U^{\prime} ; \Omega^{1 / 2}\right)$. Using the first projection, which is a submersion $U \circ U^{\prime} \rightarrow U$, we may pull back $P$ to a generalized function with pseudodifferential singularities on $V \circ U^{\prime}$; multiplying it by $f$, we obtain that $P \star f \in \mathcal{P}_{\mathrm{c}}\left(U \circ U^{\prime}, V \circ U^{\prime} ; \Omega^{1 / 2}\right)$. In the same way, we construct $f \star P \in$ $\mathcal{P}_{\mathrm{c}}\left(U^{\prime} \circ U, U^{\prime} \circ V ; \Omega^{1 / 2}\right)$.

3.2. Extending representations to pseudodifferential kernels. We fix an atlas $\mathcal{U}$. Denote by $\mathscr{E}_{u}$ the associated groupoid. 
3.2.1. Quasi-invariant measures. Recall that a measure $\mu$ on $M$ is said to be quasiinvariant by the atlas $U$ if for every bi-submersion $(U, t, s)$ adapted to the atlas $U$ the measures $\mu \circ \lambda^{s}$ and $\mu \circ \lambda^{t}$ are equivalent (here $\lambda^{s}$ and $\lambda^{t}$ are Lebesgue measures along the fibers of $s$ and $t$, respectively).

In that case, there is a measurable almost everywhere invertible section $\rho^{U}$ of $\Omega^{-1 / 2}(\operatorname{ker} d s) \otimes \Omega^{1 / 2}(\operatorname{ker} d t)$ such that for every $f \in C_{\mathrm{c}}\left(U ; \Omega^{1 / 2}(\operatorname{ker} d s) \otimes\right.$ $\left.\Omega^{1 / 2}(\operatorname{ker} d t)\right)$ we have

$$
\int_{M}\left(\int_{s^{-1}\{x\}}\left(\rho_{u}^{U}\right)^{-1} \cdot f(u)\right) d \mu(x)=\int_{M}\left(\int_{t^{-1}(x)} \rho_{u}^{U} \cdot f(u)\right) d \mu(x) .
$$

If $p:(U, t, s) \rightarrow\left(U^{\prime}, t^{\prime}, s^{\prime}\right)$ is a morphism of bi-submersions, there is a canonical isomorphism of the bundles $\Omega^{-1 / 2}(\operatorname{ker} d s) \otimes \Omega^{1 / 2}(\operatorname{ker} d t)$ and $p^{*}\left(\Omega^{-1 / 2}\left(\operatorname{ker} d s^{\prime}\right) \otimes\right.$ $\Omega^{1 / 2}\left(\operatorname{ker} d t^{\prime}\right)$ ). Under this isomorphism, the maps $\rho^{U}$ and $p^{*}\left(\rho^{U^{\prime}}\right)$ coincide (almost everywhere). We interpret this by saying that $\rho$ is defined on the groupoid $\mathscr{E}_{u}$. Furthermore, one may see that it is naturally a homomorphism.

Remark 3.2. The morphism $\rho$ defined here takes also into account the RadonNykodym derivative $\left(D^{U}\right)^{1 / 2}$ that we used in [1], $\S 5$.

Remark 3.3 (On measurable functions on $\mathscr{E}_{U}$ ). A function $f$ on $\mathscr{E}_{U}$ is just a function $f \circ \zeta$ on $\bigsqcup_{i \in I} U_{i}$ which is constant on the equivalence classes. We will say that the function $f$ is measurable with respect to the quasi-invariant measure $\mu$ if $f \circ \zeta_{U}$ is measurable (with respect to the measures $\mu \circ \lambda$ ).

Lemma 3.4. Let $(W, t, s)$ and $\left(W_{i}, t_{i}, s_{i}\right)$ be bi-submersions, $Y \subset W$ a submanifold and let $p^{i}: W \rightarrow W_{i}$ be morphisms of bi-submersions which are submersions transverse to $Y$ (here $i \in\{1,2\}$ ). Let $\mu$ be a quasi-invariant measure on $M$ and $\beta$ a measurable bounded function on $\mathcal{E}_{u}$.

For $Q \in \mathcal{P}_{\mathrm{c}}\left(W, Y ; \Omega^{1 / 2}\right)$, we have

$$
\begin{aligned}
\int_{M}\left(\int_{t_{1}^{-1}\{x\}} \rho_{w}^{W_{1}} \cdot p_{!}^{1}(Q)(w) \beta \circ \zeta_{W_{1}}(w)\right) d \mu(x) \\
=\int_{M}\left(\int_{t_{2}^{-1}\{x\}} \rho_{w}^{W_{2}} \cdot p_{!}^{2}(Q)(w) \beta \circ \zeta_{W_{2}}(w)\right) d \mu(x)
\end{aligned}
$$

Note that, by the transversality assumption, the $p_{!}^{i}(Q)$ are smooth functions. This explains the meaning of this formula.

Proof. For $Q \in C_{\mathrm{c}}^{\infty}\left(W ; \Omega^{1 / 2}\right)$ these two expressions coincide (by Fubini) with

$$
\int_{M}\left(\int_{t^{-1}\{x\}} \rho_{u}^{W} \cdot Q(u) \beta \circ \zeta_{U}(u)\right) d \mu(x) .
$$

The lemma follows from §1.2.3. 
We will use an immediate generalization of this lemma:

Lemma 3.5. Let $(W, t, s)$ and $\left(W_{i}, t_{i}, s_{i}\right)_{i \in I}$ be bi-submersions and $Y \subset W$ a closed submanifold. Assume that there is an open cover $\left(Z_{i}\right)_{i \in I}$ of $W$ and $p^{i}: Z_{i} \rightarrow W_{i}$ morphisms of bi-submersions which are submersions transverse to $Y \cap Z_{i}$. Let $\left(\chi_{i}\right)$ be a smooth partition of the identity adapted to the cover $Z_{i}$. Let $\mu$ be a quasi-invariant measure on $M$ and $\beta$ a measurable bounded function on $\mathcal{E}_{u}$.

For $Q \in \mathcal{P}_{\mathrm{c}}\left(W, Y ; \Omega^{1 / 2}\right)$, the quantity

$$
\left.\int_{M} \sum_{i \in I}\left(\int_{t_{i}^{-1}\{x\}} \rho_{w}^{W_{1}} \cdot p_{!}^{i}\left(\chi_{i} Q\right)(w) \beta \circ \zeta_{W_{i}}(w)\right) d \mu(x)\right)
$$

does not depend on the choices of I and the family $\left(Z_{i}, \chi_{i}, W_{i}, p_{i}\right)_{i \in I}$ with the above requirements.

Let $(W, t, s)$ be a bi-submersion and $Y \subset W$ a submanifold. We say that $Y$ is transverse to $\zeta_{W}$ if there is an open cover $\left(Z_{i}\right)_{i \in I}$ of $W$ and $p^{i}: Z_{i} \rightarrow W_{i}$ morphisms of bi-submersions which are submersions transverse to $Y \cap Z_{i}$. The above lemma makes sense of

$$
\int_{M}\left(\int_{t^{-1}\{x\}} \rho_{u}^{W} \cdot Q(u) \beta \circ \zeta_{U}(u)\right) d \mu(x)
$$

for $Q \in \mathcal{P}_{\mathrm{c}}\left(W, Y ; \Omega^{1 / 2}\right)$.

3.2.2. Extension of representations. We now fix an atlas $\mathcal{U}$ and a non degenerate *-representation $\Pi$ of $C^{*}(\mathcal{U})$ on a Hilbert space $\mathcal{H}$.

Let us fix some notation:

Denote by $\theta: \mathcal{A} u \rightarrow C^{*}(\mathcal{U})$ the natural morphism from $\mathcal{A} u$ to its Hausdorff completion. If $U$ is a bi-submersion adapted to $U$, denote by $\theta_{U}: C_{\mathrm{c}}^{\infty}\left(U ; \Omega^{1 / 2}\right) \rightarrow$ $C^{*}(U)$ the composition $C_{\mathrm{c}}^{\infty}\left(U ; \Omega^{1 / 2}\right) \stackrel{Q_{U}}{\longrightarrow} \mathcal{A} u \stackrel{\theta}{\rightarrow} C^{*}(U)$. Finally, put $\Pi_{U}=$ $\Pi \circ \theta_{U}$.

According to [1], $\S 5$, there is a triple $(\mu, H, \pi)$ where

(a) $\mu$ is a quasi-invariant measure on $M$,

(b) $H=\left(H_{x}\right)_{x \in M}$ is a measurable (with respect to $\mu$ ) field of Hilbert spaces over $M$,

(c) for every bi-submersion $(U, t, s)$ adapted to $U, \pi^{U}$ is a measurable (with respect to $\mu \circ \lambda$ ) section of the field of unitaries $\pi_{u}^{U}: H_{s(u)} \rightarrow H_{s(u)}$.

Moreover:

(d) $\pi$ is 'defined on $\mathcal{E}_{u}$ ':

if $f: U \rightarrow V$ is a morphism of bi-submersions, for almost all $u \in U$ we have $\pi_{u}^{U}=\pi_{f(u)}^{V}$. 
(e) $\pi$ is a homomorphism:

if $U$ and $V$ are bi-submersions adapted to $U$, we have $\pi_{(u, v)}^{U \circ V}=\pi_{u}^{U} \pi_{v}^{V}$ for almost all $(u, v) \in U \circ V$.

Then $\mathscr{H}=\int_{M}^{\oplus} H_{x} d \mu(x)$ is the space of square-integrable sections of $H$. For every bi-submersion $(U, t, s)$ adapted to $\mathcal{U}$, we have:

$$
\Pi_{U}(f)(\xi)(x)=\int_{t^{-1}\{x\}}\left(\rho_{u}^{U} \cdot f(u)\right) \pi_{u}^{U}\left(\xi_{s(u)}\right)
$$

$\mu$-a.e. for every $\xi \in \mathscr{H}$ and $x \in M$.

It follows that $\left\langle\eta, \Pi_{U}(f) \xi\right\rangle=\int_{M}\left(\int_{t^{-1}\{x\}}\left(\rho_{u}^{U} \cdot f(u)\right)\left\langle\eta_{x}, \pi_{u}^{U}(\xi)\right\rangle\right) d \mu(x)$.

Proposition 3.6. Let $(W, t, s)$ be a bi-submersion and $Y \subset W$ a submanifold transverse to $\zeta_{W}$. There is a linear map $\Pi_{W, Y}: \mathcal{P}_{\mathrm{c}}\left(W, Y ; \Omega^{1 / 2}\right) \rightarrow \mathscr{L}(H)$ such that for every open subset $Z \subset W$, every morphism $p: Z \rightarrow U$ of bi-submersions which is a submersion transverse to $Y \cap Z$, and every $Q \in \mathcal{P}_{\mathrm{c}}\left(Z, Y \cap Z ; \Omega^{1 / 2}\right) \subset$ $\mathcal{P}_{\mathrm{c}}\left(W, Y ; \Omega^{1 / 2}\right)$ we have $\Pi_{W, Y}(Q)=\Pi_{U}\left(p_{!}(Q)\right)$.

Proof. Let $\xi, \eta \in \int^{\oplus} H_{x} d \mu(x)=\mathscr{H}$ be bounded square-integrable sections, and define a bounded measurable function $\beta$ on $\mathcal{E}_{u}$ by putting $\beta \circ \zeta_{U}(u)=\left\langle\eta_{x}, \pi_{u}^{U}\left(\xi_{s_{U}}(u)\right)\right\rangle$. It follows from Lemma 3.5 that, for $Q \in \mathcal{P}_{\mathrm{c}}\left(W, Y ; \Omega^{1 / 2}\right)$, using any partition of unit adapted to a nice cover of $W$ to construct $\Pi_{W, Y}(Q)$ we have

$$
\left\langle\eta, \Pi_{W, Y}(Q) \xi\right\rangle=\int_{M}\left(\int_{t^{-1}\{x\}} \rho_{u}^{W} \cdot Q(u) \beta \circ \zeta_{U}(u)\right) d \mu(x),
$$

which is well defined by Lemma 3.5. The conclusion follows by density of bounded sections in $\mathscr{H}$.

Taking $\Pi$ to be faithful, we find:

Corollary 3.7. Let $(W, t, s)$ be a bi-submersion and $Y \subset W$ a submanifold transverse to $\zeta_{W}$. There is a linear map $\theta_{W, Y}: \mathcal{P}_{\mathrm{c}}\left(W, Y ; \Omega^{1 / 2}\right) \rightarrow \theta(\mathcal{A}(\mathcal{U}))$ such that for every open subset $Z \subset W$, every morphism $p: Z \rightarrow U$ of bi-submersions which is a submersion transverse to $Y \cap Z$ and every $Q \in \mathcal{P}_{\mathrm{c}}\left(Z, Y \cap Z ; \Omega^{1 / 2}\right) \subset \mathcal{P}_{\mathrm{c}}\left(W, Y ; \Omega^{1 / 2}\right)$ we have $\theta_{W, Y}(Q)=\theta_{U}\left(p_{!}(Q)\right)$.

3.3. Pseudodifferential kernels and multipliers. Let $(U, t, s)$ be a bi-submersion and $V \subset U$ an identity bisection. Let $U^{\prime}$ and $U^{\prime \prime}$ be bi-submersions. For $P \in$ $\mathcal{P}_{\mathrm{c}}\left(U, V ; \Omega^{1 / 2}\right), f \in C_{\mathrm{c}}^{\infty}\left(U^{\prime} ; \Omega^{1 / 2}\right)$ and $g \in C_{\mathrm{c}}^{\infty}\left(U^{\prime \prime} ; \Omega^{1 / 2}\right)$, we defined $P \star f \in$ $\mathcal{P}_{\mathrm{c}}\left(U \circ U^{\prime}, V \circ U^{\prime} ; \Omega^{1 / 2}\right)$ and $g \star P \in \mathcal{P}_{\mathrm{c}}\left(U^{\prime \prime} \circ U, U^{\prime \prime} \circ V ; \Omega^{1 / 2}\right)$ (see Definition 3.1).

Note that $V \circ U^{\prime}$ and $U^{\prime \prime} \circ V$ are bi-submersions and therefore transverse to $\zeta_{U \circ U^{\prime}}$ and $\zeta_{U^{\prime \prime} \circ U}$, respectively. 
Theorem 3.8. Let $(U, t, s)$ be a bi-submersion and $V \subset U$ an identity bisection. Let $U^{\prime}$ and $U^{\prime \prime}$ be bi-submersions. Then we have $\theta_{U^{\prime \prime}}(g) \theta_{U \circ U^{\prime}, V \circ U^{\prime}}(P \star f)=$ $\theta_{U^{\prime \prime} \circ U, U^{\prime \prime} \circ V}(g \star P) \theta_{U^{\prime}}(f)$ for $P \in \mathcal{P}_{\mathrm{c}}\left(U, V ; \Omega^{1 / 2}\right), f \in C_{\mathrm{c}}^{\infty}\left(U^{\prime} ; \Omega^{1 / 2}\right)$ and $g \in$ $C_{\mathrm{c}}^{\infty}\left(U^{\prime \prime} ; \Omega^{1 / 2}\right)$. In other words, there is a multiplier $\tilde{\theta}_{U, V}(P)$ of $\theta(\mathcal{A}(U))$ such that $\tilde{\theta}_{U, V}(P) \theta_{U^{\prime}}(f)=\theta_{U \circ U^{\prime}, V \circ U^{\prime}}(P \star f)$ and $\theta_{U^{\prime \prime}}(g) \tilde{\theta}_{U, V}(P)=\theta_{U^{\prime \prime} \circ U, U^{\prime \prime} \circ V}(g \star P)$.

Proof. It is enough to prove this theorem for $P, f, g$ with small enough support so that we may assume that there exist morphisms of bi-submersions $U \circ U^{\prime} \rightarrow W^{\prime}$ and $U^{\prime \prime} \circ U \rightarrow W^{\prime \prime}$ which are submersions respectively transverse to $V \circ U^{\prime}$ and $U^{\prime \prime} \circ V$. Then the morphisms id $\times p:\left(u^{\prime \prime}, u^{\prime}, u^{\prime}\right) \mapsto\left(u^{\prime \prime}, p\left(u, u^{\prime}\right)\right)$ and $q \times$ id are morphisms of bi-submersions and are submersions respectively transverse to $U^{\prime \prime} \circ V \circ U^{\prime}$.

We have

$$
\begin{aligned}
\theta_{U^{\prime \prime}}(g) \theta_{U \circ U^{\prime}, V \circ U^{\prime}}(P \star f) & =\theta_{U^{\prime \prime} \circ W^{\prime}}((\mathrm{id} \times p)(g \star P \star f)) \\
& =\theta_{U^{\prime \prime} \circ U \circ U^{\prime}, U^{\prime \prime} \circ V \circ U^{\prime}}(g \star P \star f)
\end{aligned}
$$

by Corollary 3.7. In the same way,

$$
\theta_{U^{\prime \prime} \circ U, U^{\prime \prime} \circ V}(g \star P) \theta_{U^{\prime}}(f)=\theta_{U^{\prime \prime} \circ U \circ U^{\prime}, U^{\prime \prime} \circ V \circ U^{\prime}}(g \star P \star f) .
$$

Note that $\tilde{\theta}_{U, V}(P)$ is a closable multiplier since its adjoint contains $\tilde{\theta}_{U^{-1}, V}\left(P^{*}\right)$ and is therefore densely defined.

Let $p: U^{\prime} \rightarrow U$ be a submersion and a morphism of bi-submersions such that $p\left(V^{\prime}\right) \subset V$. Recall from Prop. 1.7 that there is a natural map $p_{!}: \mathcal{P}\left(U^{\prime}, V^{\prime} ; \Omega^{1 / 2}\right) \rightarrow$ $\mathcal{P}\left(U, V ; \Omega^{1 / 2}\right)$ obtained by integration along fibers.

Proposition 3.9. With the above notation, we have $\tilde{\theta}_{U, V} \circ p_{!}=\tilde{\theta}_{U^{\prime}, V^{\prime}}$.

3.4. The space of pseudodifferential multipliers. We have fixed an atlas $\boldsymbol{u}=$ $\left(U_{i}, t_{i}, s_{i}\right)_{i \in I}$ together with identity bisections $V_{i} \subset U_{i}$ such that $\bigcup_{i \in I} s_{i}\left(V_{i}\right)=M .^{2}$ Denote by $\tilde{U}$ the disjoint union $\bigsqcup_{i \in I} U_{i}$ and by $\tilde{V} \subset \tilde{U}$ the disjoint union $\bigsqcup_{i \in I} V_{i}$.

Definition 3.10. Let $m \in \mathbb{Z}$. We form a space $\Psi_{\mathrm{c}}^{m}(\mathcal{U}, \mathcal{V})$. This is the image in the multiplier algebra of $\theta(\mathcal{A}(\mathcal{U}))$ of $\bigoplus_{i \in I} \mathcal{P}_{\mathrm{c}}^{m}\left(U_{i}, V_{i} ; \Omega^{1 / 2}\right)=\mathscr{P}_{\mathrm{c}}^{m}\left(\tilde{U}, \tilde{V} ; \Omega^{1 / 2}\right)$.

We define the space of pseudodifferential multipliers to be the union $\Psi_{\mathrm{c}}^{\infty}(\mathcal{U}, \mathcal{V})$ of $\Psi_{\mathrm{c}}^{m}(U, \mathcal{V})$.

An element in $\bigcap_{m \in \mathbb{Z}} \Psi_{\mathrm{c}}^{m}(\mathcal{U}, \mathcal{V})$ is called regularizing.

Let $(U, t, s)$ be a bi-submersion adapted to $U$ and $V$ an identity bisection in $U$. We just constructed a linear map $\tilde{\theta}_{U, V}: \mathcal{P}_{\mathrm{c}}\left(U, V ; \Omega^{1 / 2}\right) \rightarrow \Psi_{\mathrm{c}}^{\infty}(\mathcal{U}, \mathcal{V})$.

Elements of $\mathcal{A}(\mathcal{U})$ give obviously rise to regularizing operators. On the other hand, a regularizing operator is for every $k$ the image of a function $f_{k} \in C_{\mathrm{c}}^{k}\left(\tilde{U} ; \Omega^{1 / 2}\right)$; as the map $\theta$ is not injective, it is not clear whether $f_{k}$ can be taken constant (i.e., in $\mathcal{A}(U))$.

\footnotetext{
${ }^{2}$ For a given $i, V_{i}$ may be empty.
} 


\subsection{The longitudinal principal symbol}

Definition 3.11. The longitudinal principal symbol of an element of $\mathcal{P}_{c}^{m}\left(\tilde{U}, \tilde{V} ; \Omega^{1 / 2}\right)$ is a homogeneous function on the space $\mathscr{F}^{*} \backslash M$ of non-zero elements in $\mathcal{F}^{*}$.

To construct it, we may assume that $s_{i}: V_{i} \rightarrow M$ are injective. Identify $V_{i}$ with its image in $M$. Let $P_{i} \in \mathcal{P}\left(U_{i}, V_{i} ; \Omega^{1 / 2}\right)$ of order $m$. The principal symbol $\tilde{\sigma}_{m}\left(P_{i}\right)$ is defined to be the restriction to the subspace $\mathcal{F}^{*} \backslash M$ of $N^{*} \backslash V$ of the principal symbol of $P_{i}$. Extending it by linearity, we define the longitudinal principal symbol of any element of $\bigoplus_{i \in I} \mathcal{P}_{\mathrm{c}}^{m}\left(U_{i}, V_{i} ; \Omega^{1 / 2}\right)$.

Remark 3.12. It is not obvious whether the longitudinal principal symbol is defined in the image $\Psi_{\mathrm{c}}^{m}(\mathcal{U}, \mathcal{V})$. This happens if the groupoid $\mathscr{E}_{\mathcal{U}}$ is longitudinally smooth. Then one may define $\tilde{\sigma}_{m}(P)(x, \xi)$ using the regular representation on $L^{2}\left(\left(\mathscr{G}_{u}\right)_{x}\right)$ and a formula as in Proposition 1.4: $\tilde{\sigma}_{m}(x, \xi)=\lim _{n \rightarrow+\infty}\left\langle e^{i n \varphi} \chi_{n},(i n)^{-m} P\left(e^{i n \varphi} \chi\right)(x)\right\rangle$, where $\varphi \in C_{\mathrm{c}}^{\infty}\left(\left(\mathscr{G}_{U}\right)_{x}\right)$ with derivative $\xi$ at $x$ and $\chi_{n}$ is a suitable function which has $L^{2}$ norm 1 and has support around $x$; it is of the form $\chi_{n}(y)=n^{k / 2} \chi\left(n\|x-y\|^{2}\right)$ where $k=\operatorname{dim}\left(\mathscr{E}_{U}\right)_{x}=\operatorname{dim} \mathscr{F}_{x}$.

Even when this longitudinal principal symbol is well defined though, it is not clear whether there is an exact sequence as in Proposition 1.4, since an element in $P \in \mathcal{P}_{\mathrm{c}}^{m}\left(U, V ; \Omega^{1 / 2}\right)$ whose longitudinal principal symbol vanishes on $\mathcal{F}^{*} \subset N^{*}$ may not be in $\mathcal{P}_{\mathrm{c}}^{m-1}\left(U, V ; \Omega^{1 / 2}\right)$. Namely, it is not clear to us whether there exists $Q \in \mathcal{P}_{\mathrm{c}}^{m-1}\left(U, V ; \Omega^{1 / 2}\right)$ which has the same image in $\Psi_{\mathrm{c}}^{\infty}(U, \mathcal{V})$ as $P$. Here is an example of this situation: Consider the foliation defined by the action of $\mathrm{SO}(3)$ in $\mathbb{R}^{3}$ (Example 2.11) and take the order 0 symbol $a(x, \xi)=e^{-\frac{1}{\langle x \mid \xi\rangle^{2}}}$ outside $\mathscr{F}^{*}$ and zero in $\mathscr{F}^{*}$. This is a symbol of order 0 which vanishes on $\mathscr{F}^{*}$, but there is no pseudodifferential operator of order -1 whose symbol is $a$ in a neighborhood of $\mathscr{F}^{*}$.

Remark 3.13. If we change atlases, the pseudodifferential operators do not really change. Indeed, let $(U, t, s)$ be a bi-submersion and $V$ an identity bisection. Let $U$ be any atlas (e.g., the minimal one). Then, since $U$ carries the identity bisection, there is a neighborhood $U^{\prime}$ of $V$ in $U$ such that $\left(U^{\prime}, t, s\right)$ is adapted to $U$. Thus, there is an open cover $\left(U_{i}^{\prime}\right)$ of $U^{\prime}$ and morphisms $f_{i}: U_{i}^{\prime} \rightarrow \widetilde{U}$ such that $f_{i}\left(V \cap U_{i}^{\prime}\right) \subset \widetilde{V}$ (cf. Proposition 2.5). Every element of $\mathcal{P}_{\mathrm{c}}\left(U, V ; \Omega^{1 / 2}\right)$ can be written as $P+h$ with $P \in \mathcal{P}_{\mathrm{c}}\left(U^{\prime}, V ; \Omega^{1 / 2}\right)$ and $h \in C_{\mathrm{c}}^{\infty}\left(U, \Omega^{1 / 2}\right)$. We deduce:

(a) If we change the identity bisections we do not change at all the space $\Psi_{c}^{\infty}(\mathcal{U}, \mathcal{V})$.

(b) Let $\mathcal{U}$ and $\mathcal{U}^{\prime}$ be atlases such that $\mathcal{U}$ is adapted to $\mathcal{U}^{\prime}$. We have a natural morphism $j: C^{*}(U) \rightarrow C^{*}\left(\mathcal{U}^{\prime}\right)$. If $j$ is injective, then we have an equality $\mathcal{P}\left(\mathcal{U}^{\prime}, \mathcal{V}^{\prime}\right)=\mathcal{P}(\mathcal{U}, \mathcal{V})+\theta\left(\mathcal{A}\left(\mathcal{U}^{\prime}\right)\right)$.

\subsection{Convolution: the algebra of pseudodifferential kernels}

Lemma 3.14. Let $U, W$ be bi-submersions adapted to $U, V \subset U$ an identity bisection and $p: U \circ U \rightarrow W$ a morphism of bi-submersions which is a submersion 
strictly transverse to $V \circ U$ and to $U \circ V$. Then, for $Q_{1}, Q_{2} \in \mathcal{P}_{\mathrm{c}}\left(U, V ; \Omega^{1 / 2}\right)$ we have $\tilde{\theta}\left(Q_{1}\right) \tilde{\theta}\left(Q_{2}\right)=\tilde{\theta}\left(p_{!}\left(Q_{1} \star Q_{2}\right)\right)$.

Note that, by Proposition 1.10, $Q_{1} \star Q_{2}$ makes sense as a distribution and $p_{!}\left(Q_{1} \star Q_{2}\right)$ is pseudodifferential.

Proof. Let $U^{\prime}$ be another bi-submersion and $f \in C_{\mathrm{c}}^{\infty}\left(U^{\prime} ; \Omega^{1 / 2}\right)$. We have to show that $\tilde{\theta}\left(Q_{1}\right) \tilde{\theta}\left(Q_{2}\right) \theta(f)=\tilde{\theta}\left(p_{!}\left(Q_{1} \star Q_{2}\right)\right) \theta(f)$. To this end, we may take a faithful representation $\Pi$ and compute both $\left\langle\eta, \Pi\left(\tilde{\theta}\left(Q_{1}\right) \tilde{\theta}\left(Q_{2}\right) \theta(f)\right) \xi\right\rangle$ as well as $\left\langle\eta, \Pi\left(\tilde{\theta}\left(p_{!}\left(Q_{1} \star Q_{2}\right)\right) \theta(f)\right) \xi\right\rangle$. These two expressions are equal when $Q_{1}$ and $Q_{2}$ are smooth functions. The general case follows using §1.2.3.

Theorem 3.15. The space $\Psi_{\mathrm{c}}^{\infty}(U, \mathcal{V})$ is a subalgebra of the multiplier algebra of $\theta(\mathcal{A}(\mathcal{U}))$. More precisely, given $P_{i} \in \mathcal{P}_{\mathrm{c}}^{m_{i}}\left(\widetilde{U}, \widetilde{V} ; \Omega^{1 / 2}\right), i=1,2$, there is $P \in$ $\mathcal{P}_{\mathrm{c}}^{m}\left(\tilde{U}, \tilde{V} ; \Omega^{1 / 2}\right)$ such that $\tilde{\theta}\left(P_{1}\right) \tilde{\theta}\left(P_{2}\right)=\tilde{\theta}(P)$ and $\tilde{\sigma}_{m_{1}}\left(P_{1}\right) \tilde{\sigma}_{m_{2}}\left(P_{2}\right)=\tilde{\sigma}_{m}(P)$.

Proof. By Proposition 2.7, there is a cover of $M$ by (open) sets $s\left(V_{i}^{\prime}\right)$ where $V_{i}^{\prime}$ is an identity bisection of a bi-submersion $U_{i}^{\prime}$ adapted to $U$ for which there is a morphism of bi-submersions $p_{i}^{\prime}: U_{i}^{\prime} \circ U_{i}^{\prime} \rightarrow W_{i}$ which is a submersion strictly transverse to $V_{i}^{\prime} \circ U_{i}^{\prime}$ and to $U_{i}^{\prime} \circ V_{i}^{\prime}$.

There is a finite open cover $\left(U_{j}^{\prime \prime}\right)$ of the $\operatorname{Supp}\left(P_{1}\right) \cup \operatorname{Supp}\left(P_{2}\right)$ such that, putting $V_{j}^{\prime \prime}=U_{j}^{\prime \prime} \cap \tilde{V}$ if $s\left(V_{j}^{\prime \prime}\right) \cap s\left(V_{k}^{\prime \prime}\right) \neq \emptyset$, there are morphisms of bi-submersions from $U_{j}^{\prime \prime}$ and from $U_{k}^{\prime \prime}$ to the same $U_{i}^{\prime}$ which are submersions.

Using a partition of the identity adapted to $U_{j}^{\prime \prime}$, we are reduced to the case where $P_{1} \in \mathcal{P}_{\mathrm{c}}^{m_{1}}\left(U_{j}^{\prime \prime}, V_{j}^{\prime \prime} ; \Omega^{1 / 2}\right)$ and $P_{2} \in \mathcal{P}_{\mathrm{c}}^{m_{2}}\left(U_{k}^{\prime \prime}, V_{k}^{\prime \prime} ; \Omega^{1 / 2}\right)$.

If $V_{j}^{\prime \prime} \circ V_{k}^{\prime \prime}=\emptyset$ then $P_{1} \star P_{2} \in \mathcal{P}_{\mathrm{c}}\left(U_{j}^{\prime \prime} \circ U_{k}^{\prime \prime}, W ; \Omega^{1 / 2}\right)$ with $W=V_{j}^{\prime \prime} \circ U_{k}^{\prime \prime} \cup$ $U_{j}^{\prime \prime} \circ V_{k}^{\prime \prime}$, and therefore $\tilde{\theta}\left(P_{1}\right) \tilde{\theta}\left(P_{2}\right) \in \theta(\mathcal{A} u)$.

If $s\left(V_{j}^{\prime \prime}\right) \cap s\left(V_{k}^{\prime \prime}\right) \neq \emptyset$, we may replace $P_{1}$ and $P_{2}$ by their images $Q_{1}, Q_{2}$ in $\mathcal{P}_{\mathrm{c}}\left(U_{i}^{\prime}, V_{i}^{\prime} ; \Omega^{1 / 2}\right)$ (Proposition 3.9). Now, by Proposition 1.10, $Q_{1} \star Q_{2}$ makes sense as a distribution and $\left(p_{i}^{\prime}\right) !\left(Q_{1} \star Q_{2}\right)$ is pseudodifferntial and has the right longitudinal principal symbol. The result follows by Lemma 3.14.

\section{Longitudinal ellipticity}

In this section we assume that the manifold $M$ is compact.

Definition 4.1. A pseudodifferential operator $P \in \mathcal{P}_{\mathrm{c}}^{m}(U, \mathcal{V})$ is said to be longitudinally elliptic if its longitudinal principal symbol $\tilde{\sigma}_{m}(P)$ is invertible. 
4.1. Parametrix. We now state the analogues in our setting of some most important classical results in the pseudodifferental calculus.

Theorem 4.2 (Existence of quasi-inverses). Let $P \in \mathcal{P}_{\mathrm{c}}^{m}\left(\tilde{U}, \tilde{V} ; \Omega^{1 / 2}\right)$ be a longitudinally elliptic operator of order $m$. There is a pseudodifferential operator $Q \in \mathcal{P}_{\mathrm{c}}^{-m}\left(\tilde{U}, \widetilde{V} ; \Omega^{1 / 2}\right)$ of order $-m$ such that $1-\tilde{\theta}(P) \tilde{\theta}(Q)$ and $1-\tilde{\theta}(Q) \tilde{\theta}(P)$ are regularizing.

The main ingredient of the proof is:

Lemma 4.3. Let $P \in \mathcal{P}_{\mathrm{c}}^{m}\left(\tilde{U}, \tilde{V} ; \Omega^{1 / 2}\right)$ be longitudinally elliptic and $S \in \Psi_{\mathrm{c}}^{k}(\tilde{U}, \tilde{V})$. Then there exists $Q \in \mathcal{P}_{\mathrm{c}}^{k-m}\left(\tilde{U}, \tilde{V} ; \Omega^{1 / 2}\right)$ such that $\tilde{\theta}(P) \tilde{\theta}(Q)-S \in \Psi_{\mathrm{c}}^{k-1}(\tilde{U}, \tilde{V})$.

This, in turn, relies on the following result:

Lemma 4.4. Let $P \in \mathcal{P}_{\mathrm{c}}^{m}\left(\tilde{U}, \tilde{V} ; \Omega^{1 / 2}\right)$ be longitudinally elliptic. Then there exist

(i) a finite set $I$, bi-submersions $\left(U_{i}^{\prime}, t_{i}^{\prime}, s_{i}^{\prime}\right)_{i \in I}$, identity bisections $V_{i}^{\prime} \subset U_{i}^{\prime}$ and morphisms of bi-submersions $p^{i}: U_{i}^{\prime} \circ U_{i}^{\prime} \rightarrow W_{i}$ that are submersions strictly transverse to $U_{i}^{\prime} \circ V_{i}^{\prime}$ and to $V_{i}^{\prime} \circ U_{i}^{\prime}$,

(ii) open relatively compact subsets $U_{i} \subset U_{i}^{\prime}$ such that $V_{i}=V_{i}^{\prime} \cap U_{i}$ is relatively compact in $V_{i}^{\prime}$,

(iii) $\bigcup_{i \in I} s_{i}\left(V_{i}\right)=M$,

(iv) operators $P_{i}^{\prime} \in \mathcal{P}_{\mathrm{c}}^{m}\left(U_{i}^{\prime}, V_{i}^{\prime} ; \Omega^{1 / 2}\right)$ whose (plain) principal symbol $\sigma_{m}\left(P_{i}^{\prime}\right)$ is invertible on $\bar{V}_{i}$,

(v) smooth functions $\phi_{i} \in C_{c}^{\infty}\left(U_{i}^{\prime}\right)$ such that $\left.\phi_{i}\right|_{V_{i}}=1$,

so that $\phi_{i} P-P_{i}^{\prime}$ is regularizing.

Proof of Lemma 4.4. Let $x \in M$; consider a pair $\left(U^{\prime}, V^{\prime}\right)$ such that $\left(U^{\prime}, t^{\prime}, s^{\prime}\right)$ is a bi-submersion, $V^{\prime}$ is an identity bisection, which is minimal at a point $v \in V^{\prime}$ with $s(v)=x$. Take $\phi \in C_{\mathrm{c}}^{\infty}(M)$ to be 1 in a neighborhood of $x$ with support in $s\left(V^{\prime}\right)$. There exists an operator $P^{\prime} \in \mathcal{P}\left(U^{\prime}, V^{\prime}\right)$ of order $m$ such that $\phi P-P^{\prime}$ is regularizing. Whence $\sigma_{m}(P) \phi=\left.\sigma_{m}\left(P^{\prime}\right)\right|_{\mathcal{F}^{*} *}$ and $\sigma_{m}\left(P^{\prime}\right)(v, \xi)$ is invertible for every $\xi \in N_{v}^{*}$ since $\left(U^{\prime}, V^{\prime}\right)$ is minimal at $v$. The set of $w \in V^{\prime}$ for which $\sigma_{m}\left(P^{\prime}\right)(w, \xi)$ is invertible for all $\xi \in N_{w}^{*}$ is open (by compactness of the spheres). It follows that $\sigma_{m}\left(P^{\prime}\right)(w, \xi)$ is invertible for every $w$ in a small enough neighborhood of $u$ in $V^{\prime}$ and $\xi \in N_{w}^{*}$.

The result follows by compactness of $M$ (using Proposition 2.7).

Here are the proofs of the previous two results:

Proof of Lemma 4.3. Consider the data $\left(U_{i}, V_{i}\right),\left(U_{i}^{\prime}, V_{i}^{\prime}\right)$ and $P_{i}^{\prime}$ of Lemma 4.4 associated to $P$. Let $\left(\chi_{i}\right)_{i \in I}$ be a partition of unity associated to the cover $\left(V_{i}\right)_{i \in I}$. 
As the (plain) symbol of $P_{i}^{\prime}$ is invertible over $\bar{V}_{i}$, there exist $T_{i} \in \mathcal{P}\left(U_{i}^{\prime}, V_{i}^{\prime} ; \Omega^{1 / 2}\right)$ of order $-m$ whose (plain) principal symbol is $\sigma_{m}\left(P_{i}^{\prime}\right)^{-1}$ in a neighborhood of $\bar{V}_{i}$. Then $P_{i}^{\prime} T_{i} \chi_{i}-\chi \in \mathcal{P}_{\mathrm{c}}^{-1}\left(U_{i}^{\prime}, V_{i}^{\prime} ; \Omega^{1 / 2}\right)$ (we use $p_{!}^{i}$ to make this composition).

There is an operator $R_{i} \in \mathcal{P}_{\mathrm{c}}^{k}\left(U_{i}, V_{i} ; \Omega^{1 / 2}\right)$ whose image in $\Psi_{\mathrm{c}}^{\infty}(\mathcal{U}, \mathcal{V})$ is $\chi_{i} S$ up to regularizing operators. Put then $Q=\sum_{i \in I} T_{i} R_{i}$.

Proof of Theorem 4.2. Lemma 4.3 allows us to follow the classical proof: First construct $Q_{0} \in \mathcal{P}_{\mathrm{c}}^{-m}\left(\tilde{U}, \tilde{V} ; \Omega^{1 / 2}\right)$ such that $I-Q_{0} P$ is of negative order. By putting $Q_{k}=Q_{0}\left(I-P Q_{0}\right)^{k}=\left(I-Q_{0} P\right)^{k} Q_{0}$ we obtain a sequence of operators of order $-m-k, i \in \mathbb{N}$. From 1.5 it follows that there exists $Q$ of order $-m$ (asymptotically the sum of the $Q_{k}$ ) such that $I-P Q$ and $I-Q P$ are regularizing.

\subsection{Square roots}

Theorem 4.5 (Square roots). If $P \in \mathcal{P}_{\mathrm{c}}^{2 m}\left(\tilde{U}, \tilde{V} ; \Omega^{1 / 2}\right)$ is self-adjoint of even order and $\tilde{\sigma}_{2 m}(P)>0$, there is a self-adjoint $Q \in \mathcal{P}_{\mathrm{c}}^{m}\left(\tilde{U}, \tilde{V} ; \Omega^{1 / 2}\right)$ such that $P-Q^{2}$ is smoothing.

Proof. We use Lemma 4.4 and the notation there. Coming back to its proof, we may assume that the (plain) symbol of $P_{i}^{\prime}$ restricted to $V_{i}$ is $>0$. Let then $Q_{i}^{\prime} \in$ $\mathcal{P}_{\mathrm{c}}^{-1}\left(U_{m}^{\prime}, V_{i}^{\prime} ; \Omega^{1 / 2}\right)$ that we may assume self-adjoint such that the restriction to $V_{i}$ of its plain principal symbol is $\sqrt{\sigma_{2 m}\left(P_{i}^{\prime}\right)}$. Let $\chi_{i}^{2}$ be a partition of the identity adapted to $V_{i}$, and put $Q_{0}=\sum_{i \in I} \chi_{i} Q_{i}^{\prime} \chi_{i}$. Then $P-Q_{0}^{2}$ is of order $2 m-1$. Note that $Q_{0}$ is elliptic.

Suppose we constructed $Q_{0}, \ldots, Q_{n-1}$ self-adjoint such that $Q_{j}$ has order $m-j$ and $R_{n}=P-\left(\sum_{j=0}^{n-1} Q_{j}\right)^{2}$ is self-adjoint of order $2 m-n$. Due to Lemma 4.3, we find $Q_{n}$ of order $m-n$ such that $2 Q_{0} Q_{n}-R_{n}$ has order $m-n-1$. It is a consequence of Proposition 1.10 that $Q_{0}$ and $Q_{n}$ commute up to lower order, therefore $2 Q_{n} Q_{0}-R_{n}$ has order $m-n-1$. Since $Q_{0}$ and $R_{n}$ are self-adjoint, we may replace $Q_{n}$ by $1 / 2\left(Q_{n}+Q_{n}^{*}\right)$. Hence $Q_{n}$ is a sequence that satisfies Theorem 1.5. Put $Q^{\prime}$ the asymptotic sum of the $Q_{j}$ s and $Q=\frac{Q^{\prime}+Q^{\prime *}}{2}$. This is self-adjoint and also an asymptotic sum for $Q_{n}$. By construction, $P-Q^{2}$ is smoothing.

\section{The extension of zero-order pseudodifferential operators}

Let us begin by a remark that will allow us to assume that the manifold $M$ is compact.

Remark 5.1. (a) Let $M^{\prime}$ be an open subset of $M$. Then $C_{\mathrm{c}}^{\infty}\left(M^{\prime}\right) \mathcal{F}$ is a foliation $\mathcal{F}^{\prime}$ on $M^{\prime}$. A bi-submersion $(U, t, s)$ for $(M, \mathcal{F})$ restricts to a bi-submersion of $\left(M^{\prime}, \mathcal{F}^{\prime}\right)$ by putting $U^{\prime}=\left\{u \in U ; s(u) \in M^{\prime}, t(u) \in M^{\prime}\right\}$. In this way, an atlas $U$ of $(M, \mathcal{F})$ restricts to an atlas of $U^{\prime}$ of $\left(M^{\prime}, \widetilde{F}^{\prime}\right)$. By extending compactly supported functions on $U^{\prime}$, we embed $C^{*}\left(\mathcal{U}^{\prime}\right)$ into $C^{*}(\mathcal{U})$. 
(b) Assume $M^{\prime}$ is relatively compact in $M$. Then there exists $f \in C_{\mathrm{c}}^{\infty}(M)$ which is everywhere nonzero on $M^{\prime}$. Note that $\{f X ; X \in \mathcal{F}\}$ is a foliation on $M$ which has the same restriction to $M^{\prime}$ as $M$. There is a compact manifold $M^{\prime \prime}$ which contains an open subset diffeomorphic to a neighborhood of the support of $f$. Then $M^{\prime \prime}$ carries a foliation which has the same restriction to $M^{\prime}$ as $\mathcal{F}$.

Lemma 5.2. Every sufficiently negative order pseudodifferential operator defines an element in $C^{*}(U)$. More precisely, given a bi-submersion $(U, t, s)$ and an identity bisection $V \subset U$, let $P \in \mathcal{P}_{\mathrm{c}}^{-m}\left(U, V ; \Omega^{1 / 2}\right)$ with $m$ strictly bigger than the dimension of the fibers of $s$ and $t$, then $\tilde{\theta}(P) \in C^{*}(\mathcal{U})$.

Proof. A continuous function with compact support in $U$ defines an element in $C^{*}(U)$ (thanks to the $L^{1}$ estimate and by density of $C_{\mathrm{c}}^{\infty}(U)$ ). Now if $a$ is of sufficiently negative order, the integral

$$
\iint_{N^{*} v} a(v, \xi) \chi(u) e^{i\langle u, \xi\rangle}
$$

makes sense and thus the distribution $P$ is actually a continuous function with compact support in $U$.

Theorem 5.3. (a) Negative order pseudodifferential operators are in $C^{*}(\mathcal{U})$, as well as those zero-order operators whose principal symbol vanishes on $\mathcal{F}^{*}$.

(b) Zero-order pseudodifferential operators define bounded multipliers of the $C^{*}$ algebra of the foliation.

Proof. Using Remark 5.1, we may assume $M$ is compact. By Theorem 4.5, if $\left\|\tilde{\sigma}_{P}^{0}\right\|<t$, there is $Q \in \Psi_{\mathrm{c}}^{\infty}(\mathcal{U}, \mathcal{V})$ such that $P^{*} P+Q^{*} Q=t^{2}+R$, where $R$ is of negative enough order, so that it belongs to the $\mathrm{C}^{*}$-algebra of the foliation (in fact it can even be taken smoothing). We have $(P f)^{*}(P f)+(Q f)^{*}(Q f)=t^{2} f^{*} f+f^{*} R f$ for all $f \in \mathcal{A}(U)$.

It follows that

- $\|P f\| \leq k\|f\|$, where $k=\sqrt{t^{2}+\|R\|}$, hence $P$ extends to a bounded multiplier and (b) follows;

- if $\sigma_{P}^{0}=0$, then in the quotient $\mathrm{C}^{*}$-algebra $\overline{\Psi_{\mathrm{c}}^{\infty}(\mathcal{U}, \mathcal{V})} / C^{*}(\mathcal{U})$, the norm of $P$ is $\leq t$ for all $t>0$, whence $P \in C^{*}(\mathcal{U})$.

We thus have an exact sequence of $\mathrm{C}^{*}$-algebras

$$
0 \rightarrow C^{*}(M, \mathcal{F}) \rightarrow \Psi^{*}(M, \mathcal{F}) \rightarrow B \rightarrow 0,
$$

where $\Psi^{*}(M, \mathcal{F})$ denotes the closure of the algebra of zero-order pseudodifferential operators with respect to multiplier norm and order 0 symbol. The algebra $B$ is a quotient of the algebra $C_{0}\left(S^{*} \mathcal{F}\right)$ of continuous functions on the cosphere "bundle". As discussed in Remark 3.12, if the groupoid $\mathscr{E}_{U}$ is longitudinally smooth, then $B=C_{0}\left(S^{*} \mathcal{F}\right)$. 


\section{Longitudinally elliptic operators of positive order}

In this section we assume that $M$ is compact.

6.1. Longitudinally elliptic operators and regular multipliers. Recall [2], [3], [20] that an unbounded multiplier $T$ of a $\mathrm{C}^{*}$-algebra is said to be regular if it is densely defined, its adjoint is densely defined and its graph is orthocomplemented, which means that $A \oplus A=G \oplus G^{\perp}$, where $G=\{(x, T x): x \in \operatorname{dom} T\}$ is the graph of $T$ and $G^{\perp}=\left\{\left(T^{*} y,-y\right): y \in \operatorname{dom} T^{*}\right\}$ its orthogonal complement for the obvious $A$ valued scalar product in $A \oplus A$.

Let $\Pi$ be a non degenerates representation of $A$. It extends to a representation $\widetilde{\Pi}$ of the multiplier algebra $\mathcal{M}(A)$. Every regular unbounded multiplier $T$ of $A$ gives rise to a closed operator $\widehat{\Pi}(T)$ whose graph is the closure of $\{(\Pi(a) \xi, \Pi(T a) \xi): a \in$ $\left.\operatorname{dom} T, \xi \in H_{\Pi}\right\}$. The adjoint of $\widehat{\Pi}(T)$ is $\widehat{\Pi}\left(T^{*}\right)$. In particular, if $T$ is self-adjoint, so is $\widehat{\Pi}(T)$.

In [18], $\S 3-4$, Vassout proved that elliptic pseudodifferential operators (of positive order) on a Lie groupoid $G$ give rise to regular operators. The proof in [18] can be adapted to our setting to show:

Theorem 6.1. If $P \in \Psi_{\mathrm{c}}^{m}(U, \mathcal{V})$ is the image of a longitudinally elliptic operator of order $m$, then $\bar{P}$ is a regular multiplier on $C^{*}(U)(m>0)$.

Proof. For every $S \in \Psi_{\mathrm{c}}^{0}(\mathcal{U}, \mathcal{V})$, denote by $\bar{S}$ its closure which is a multiplier of $C^{*}(\mathcal{U})$.

Let $Q$ be a parametrix of $P$ and write $I-Q P=R$. Let $T \in \mathscr{L}\left(C^{*}(\mathcal{U}) \oplus\right.$ $C^{*}(U)$ ) be the (adjointable) operator of the $C^{*}$-module $C^{*}(U) \oplus C^{*}(U)$ with matrix $\left(\frac{\bar{R}}{\overline{P R}} \frac{\bar{Q}}{P Q}\right)$.

The restriction to $\theta(\mathcal{A}) \oplus \theta(\mathcal{A})$ of $T^{2}$ has matrix $\left(\begin{array}{cc}(R+Q P) R & (R+Q P) Q \\ P(R+Q P) R & P(R+Q P) Q\end{array}\right)$, whence $T^{2}=T($ as $R+Q P=I)$.

- Since $T$ is an (adjointable) idempotent element in $\mathscr{L}\left(C^{*}(U) \oplus C^{*}(\mathcal{U})\right.$ ), its range is orthocomplemented.

- Since $T$ is continuous, and $\theta(\mathcal{A}(\mathcal{U})) \oplus \theta(\mathcal{A}(\mathcal{U}))$ is dense in $C^{*}(\mathcal{U}) \oplus C^{*}(\mathcal{U})$, we deduce that the range of $T$ is the closure of $T(\theta(\mathcal{A}(\mathcal{U})) \oplus \theta(\mathcal{A}(\mathcal{U})))$

- If $(x, y) \in \theta(\mathcal{A}(\mathcal{U}))$, we find $T(x, y)=(R x+Q y, P(R x+Q y))$. If furthermore $y=P x, T(x, y)=(x, y)$. It follows that $T(\theta(\mathcal{A}(\mathcal{U})) \oplus \theta(\mathcal{A}(\mathcal{U})))$ is the graph of $P$.

We just proved that the closure of the graph of $P$ is orthocomplemented, i.e., $\bar{P}$ is regular.

Remarks 6.2. In the same way we may adapt the proofs of [18] to our setting to prove: 
(a) Any two longitudinally elliptic operators $P, P^{\prime} \in \Psi_{\mathrm{c}}^{\infty}(U, \mathcal{V})$ of the same order have the same domain.

(b) Longitudinally elliptic operators define a filtration of $C^{*}(U)$ by Sobolev modules. If $P$ is of order $k>0$ then

- $H^{k}=\operatorname{dom} P$ with scalar product $\langle\alpha, \beta\rangle_{k}=\langle P \alpha, P \beta\rangle+\langle\alpha, \beta\rangle$,

- $H^{-k}$ is the completion of $C^{*}(U)$ with norm $\|\xi\|_{-k}=\left\|\left(1+P^{*} P\right)^{-1 / 2} \xi\right\|$.

This filtration satisfies the following properties:

- If $k>k^{\prime}$ then the identity on $\mathcal{A} u$ extends to a compact morphism of Hilbert modules $i_{k, k^{\prime}}: H^{k} \hookrightarrow H^{k^{\prime}}$.

- Any $P \in \Psi_{\mathrm{c}}^{\infty}(U, \mathcal{V})$ of order $m$ defines an element of $\mathscr{L}\left(H^{k} ; H^{k-m}\right)$ for any $k$.

(c) Using the Sobolev spaces above one can define an algebra $\Psi^{-\infty}(\mathcal{U})$ of smoothing pseudodifferential operators without compact support by calling an operator $R$ smoothing iff $R \in \bigcap_{s, t \in \mathbb{R}} \mathscr{L}\left(H^{s} ; H^{t}\right)$. It follows that $\mathcal{A} u \subset \Psi^{-\infty}(U)$ is a dense subalgebra.

6.2. Application: Laplacian of a singular foliation. As a particular case we may construct a Laplacian operator for every foliation and prove that it is a positive selfadjoint operator of $L^{2}(M)$.

Let $(M, \mathcal{F})$ be a foliation. Every vector field $X \in \mathcal{F}$ defines a differential hence pseudodifferential operator $X \in \Psi_{\mathrm{c}}^{\infty}(\mathcal{U}, \mathcal{V})$ (cf. example 1.2).

Since $M$ is compact, $\mathscr{F}$ is generated by finitely many vector fields $X_{1}, \ldots, X_{N}$.

Definition 6.3. The element $\Delta=\sum_{k=1}^{N} X_{k}^{*} X_{k}$ is called a Laplacian of the foliation $\mathcal{F}$.

From the definition of $\Delta$ we have:

Theorem 6.4. A Laplacian $\Delta$ is a formally self-adjoint elliptic operator of order 2; it therefore defines a regular (unbounded) self-adjoint multiplier of $C^{*}(\mathcal{U})$.

We may apply that to the natural representation of $C^{*}(U)$ on $L^{2}(M)$ (which can be seen as the integration of the trivial representation $(\lambda, \mathbb{C})$ of the groupoid in the sense of [1], §5.1, where $\lambda$ is the Lebesgue measure on $M$ ).

Corollary 6.5. The Laplacian $\Delta$ defines an unbounded, self-adjoint operator $\Delta$ of $L^{2}(M)$. In other words, take vector fields $X_{1}, \ldots, X_{N}$ on a compact manifold. Assume that the module they generate is a foliation, i.e., for every $(i, j)$ there exist $f_{i, j, k} \in C^{\infty}(M)$ such that $\left[X_{i}, X_{j}\right]=\sum_{k} f_{i, j, k} X_{k}$. Then the closure in $L^{2}(M)$ of $\sum_{k=1}^{N} X_{j}^{*} X_{j}$ is self-adjoint. 
Remarks 6.6. (a) One can apply Theorem 6.4 to other natural representations of $C^{*}(\mathcal{U})$. One may for instance take the representation on $L^{2}$ of a leaf. There, the Laplacian is elliptic and the difficulty comes from the fact that the leaf may not be compact.

(b) The spectrum of the image of every regular operator - and in particular of $\Delta-$ is the same if we take two weakly equivalent representations of $C^{*}(\mathcal{U})$. This applies if we compare the representation in $L^{2}(M)$ and a representation in $L^{2}$ of a dense leaf (with some amenability assumptions).

\section{References}

[1] I. Androulidakis and G. Skandalis, The holonomy groupoid of a singular foliation. $J$. Reine Angew. Math. 626 (2009), 1-37. Zbl 1161.53020 MR 2492988

[2] S. Baaj, Multiplicateurs non bornés. Thèse, Univ. P. et M.Curie, Paris 1980.

[3] S. Baaj and P. Julg, Théorie bivariante de Kasparov et opérateurs non bornés dans les $C^{*}$-modules hilbertiens. C. R. Acad. Sci. Paris Sér. I Math. 296 (1983), 875-878. Zbl 0551.46041 MR 715325

[4] B. Bigonnet et J. Pradines, Graphe d'un feuilletage singulier, C. R. Acad. Sci. Paris Sér. I Math. 300 (1985), 439-442. Zbl 0581.57013 MR 0794018

[5] A. Connes, Sur la théorie non commutative de l'intégration. In Algèbres d'opérateurs (Sém., Les Plans-sur-Bex, 1978), Lecture Notes in Math. 725, Springer-Verlag, Berlin 1979, 19-143. Zbl 0412.46053 MR 0548112

[6] A. Connes, A survey of foliations and operator algebras. In Operator algebras and applications, Part I (Kingston, Ont., 1980), Proc. Sympos. Pure Math. 38, Amer. Math. Soc., Providence, R.I., 1982, 521-628. Zbl 0531.57023 MR 0679730

[7] C. Debord, Local integration of Lie algebroids. In Lie algebroids and related topics in differential geometry (Warsaw, 2000), Banach Center Publ. 54, Polish Acad. Sci., Warsaw 2001, 21-33. Zbl 1007.22008 MR 1881646

[8] C. Debord, Holonomy groupoids of singular foliations. J. Differential Geom. 58 (2001), 467-500. Zbl 1034.58017 MR 1906783

[9] T. Fack and G. Skandalis, Sur les représentations et idéaux de la $C^{*}$-algèbre d'un feuilletage. J. Operator Theory 8 (1982), 95-129. Zbl 0493.46051 MR 670180

[10] M. Khoshkam and G. Skandalis, Regular representation of groupoid $C^{*}$-algebras and applications to inverse semigroups. J. Reine Angew. Math. 546 (2002), 47-72. Zbl 1029.46082 MR 1900993

[11] E. C. Lance, Hilbert $C^{*}$-modules. London Math. Soc. Lecture Note Ser. 210, Cambridge University Press, Cambridge 1995. Zbl 0822.46080 MR 1325694

[12] B. Monthubert and F. Pierrot, Indice analytique et groupoïdes de Lie. C. R. Acad. Sci. Paris Sér. I Math. 325 (1997), 193-198. Zbl 0955.22004 MR 1467076

[13] V. Nistor, A. Weinstein, and P. Xu, Pseudodifferential operators on differential groupoids. Pacific J. Math. 189 (1999), 117-152. Zbl 0940.58014 MR 1687747 
[14] J. Pradines, Feuilletages: holonomie et graphes locaux. C. R. Acad. Sci. Paris Sér. I Math. 298 (1984), 297-300. Zbl 0568.57018 MR 765427

[15] J. Pradines, How to define the differentiable graph of a singular foliation. Cahiers Topologie Géom. Différentielle Catégoriques 26 (1985), 339-380. Zbl 0576.57023 MR 816646

[16] J. N. Renault, A groupoid approach to $C^{*}$-algebras. Lecture Notes in Math. 793, Springer-Verlag, Berlin 1980. Zbl 0433.46049 MR 0584266

[17] M. E. Taylor, Pseudodifferential operators. Princeton Math. Ser. 34, Princeton University Press, Princeton, N.J. 1981. Zbl 0453.47026 MR 0618463

[18] S. Vassout, Unbounded pseudodifferential calculus on Lie groupoids. J. Funct. Anal. 236 (2006), 161-200. Zbl 1105.58014 MR 2227132

[19] H. E. Winkelnkemper, The graph of a foliation. Ann. Global Anal. Geom. 1 (1983), 51-75. Zbl 0526.53039 MR 739904

[20] S. L. Woronowicz and K. Napiórkowski, Operator theory in the $C^{*}$-algebra framework. Rep. Math. Phys. 31 (1992), 353-371. Zbl 0793.46039 MR 1232646

Received September 14, 2009

I. Androulidakis, Mathematisches Institut, Universität Göttingen, Bunsenstrasse 3-5, 37073 Göttingen, Germany

E-mail: iakovos@uni-math.gwdg.de

G. Skandalis, Institut de Mathématiques de Jussieu, UMR 7586 CNRS, Université Diderot Paris 7, 175, rue du Chevaleret, 75013 Paris, France

E-mail: skandal@math.jussieu.fr 